\title{
A GENERALIZATION OF THE WIENER RATIONAL BASIS FUNCTIONS ON INFINITE INTERVALS: PART I-DERIVATION AND PROPERTIES
}

\author{
AKIL C. NARAYAN AND JAN S. HESTHAVEN
}

\begin{abstract}
We formulate and derive a generalization of an orthogonal rationalfunction basis for spectral expansions over the infinite or semi-infinite interval. The original functions, first presented by Wiener, are a mapping and weighting of the Fourier basis to the infinite interval. By identifying the Fourier series as a biorthogonal composition of Jacobi polynomials/functions, we are able to define generalized Fourier series which, when appropriately mapped to the whole real line and weighted, generalize Wiener's basis functions. It is known that the original Wiener rational functions inherit sparse Galerkin matrices for differentiation, and can utilize the fast Fourier transform (FFT) for computation of the expansion coefficients. We show that the generalized basis sets also have a sparse differentiation matrix and we discuss connection problems, which are necessary theoretical developments for application of the FFT.
\end{abstract}

\section{INTRODUCTION}

The approximation of a function by a finite sum of basis functions has long been a hallmark tool in numerical analysis. Over the finite interval much is known about expansion properties, and periodic Fourier expansions or polynomial expansions are well-studied. On infinite intervals there are complications due to the unbounded domain on which approximation is necessary. Nevertheless, many basis sets have been successfully investigated in this case; the Hermite functions provide a suitable method for approximation when it can be assumed that the function decays exponentially; for functions that do not decay exponentially, the so-called mapped Chebyshev rational functions fill the void and open up the possibility for utilizing the fast Fourier transform (FFT); additionally, a Fourier basis mapped to the real line has been explored and provides an additional method for function approximation over the infinite interval. This last basis set serves as an inspiration for the family of basis sets proposed in this paper.

Despite the use of available methods for function approximation over the infinite interval, there are known shortcomings. The Hermite functions/polynomials do not admit an FFT exploitation and have problems approximating functions that do not decay exponentially (which is to say, most functions). However, the solutions to differential equations by Hermite approximations have been relatively successful and in some cases yield superior results when compared to a Chebyshev (mapped

Received by the editor May 28, 2009 and, in revised form, April 11, 2010.

2010 Mathematics Subject Classification. Primary 65D15, 41A20, 42A10.

Key words and phrases. Spectral methods, infinite interval, rational functions.

(C)2010 American Mathematical Society 
or truncated) approximation [5]. The Whittaker cardinal interpolant functions [26], or Sinc functions, provide a remarkably simple method to approximate a function with known equispaced evaluations. The drawback is a relatively small class of functions for which such an expansion is complete. However, the ease of applying Sinc methods has led to a great number of applications [17. The Chebyshev rational functions [4], 8] are robust with respect to the deficiencies of the Hermite and Sinc bases, but they have some disadvantages compared with the generalized Wiener basis that we will derive.

On the semi-infinite interval Laguerre polynomial/function expansions are the classical approximation technique [1, but these techniques suffer from the same problems as Hermite expansions. An alternative technique involves mapping Jacobi polynomials to the infinite interval [6]. This mapping technique makes it possible to accurately approximate algebraically-decaying functions on the semiinfinite interval, but introduces additional computational issues for the solution to differential equations. The generalized Wiener basis can be employed on the semiinfinite interval; this results in a basis set that is also a mapped Jacobi polynomial method. However, the Wiener mapping is very different from that presented in the literature, and is an alternative to these existing techniques.

Our generalized basis is inspired by a collection of orthogonal and complete functions originally proposed by Wiener [27. He introduces the functions

$$
\phi_{n}(x)=\frac{(1-i x)^{n}}{\sqrt{\pi}(1+i x)^{n+1}}, \quad n \in \mathbb{N}_{0}=\mathbb{N} \cup\{0\}
$$

as Fourier transforms of the Laguerre functions, and he furthermore shows that these functions are orthogonal under the $L^{2}$ conjugate inner product. Higgins [15] expands this result by presenting the functions $\phi_{n}$ along with their complex conjugates as a complete system in $L^{2}$. Following this, others have used these functions by applying them to the solution of differential equations [11, 10. We note that the functions $\phi_{n}(x)$ presented above have magnitude that decays like $\frac{1}{x}$ as $|x| \rightarrow \infty$. We will generalize the above functions so that they have decay $\frac{1}{x^{s}}$ for any $s>\frac{1}{2}$. The ability to choose the rate of decay of the basis set is an advantage if such information is present about the nature of the function to be approximated (e.g. [16], [18]). Furthermore, we will show that this basis admits sparse Galerkin matrices and that the FFT can be used in certain cases to evaluate and manipulate the series.

This paper is concerned with the derivation and theoretical properties of the generalized Wiener rational function basis. Computational considerations, numerical examples, and comparisons with alternative basis sets are presented in a second part. The outline of this paper is as follows. In Section 2 we formulate and derive the basis, which stems from a generalized Fourier series. Section 3 lists the properties of the basis functions. In Section 4 we discuss how the Wiener basis set may be used to approximate functions on the semi-infinite interval. Finally, we briefly discuss the connections between the Wiener rational functions and alternative methods in Section [5 and summarize and present an outlook in Section 6 for Part II, dealing with numerical issues. 
TABLE 1. Isomorphic transforms between different domains.

\begin{tabular}{|c|c|c|c|c|}
\hline & $x$ & $z$ & $\theta$ & $r$ \\
\hline$x$ & $x \in[0, \infty]$ & $z=-\frac{x-i}{x+i}$ & $\theta=2 \arctan (x)$ & $r=\frac{1-x^{2}}{1+x^{2}}$ \\
\hline$z$ & $x=i \frac{1-z}{1+z}$ & $z \in \mathbb{T}^{+}$ & $\theta=\arg z$ & $r=\frac{1}{2}(z+\bar{z})$ \\
\hline$\theta$ & $x=\tan \left(\frac{\theta}{2}\right)$ & $z=e^{i \theta}$ & $\theta \in[0, \pi]$ & $r=\cos \theta$ \\
\hline$r$ & $x=\sqrt{\frac{1-r}{1+r}}$ & $z=e^{i \arccos r}$ & $\theta=\arccos r$ & $r \in[-1,1]$ \\
\hline
\end{tabular}

\section{DeRIVATION OF THE BASIS}

We seek $L^{2}$-orthogonal and complete basis functions whose domain is the entire real line. In addition, we desire the ability to specify a parameter $s>\frac{1}{2}$ that will denote the polynomial decay at $\pm \infty$ of each of the basis functions. Let us call these functions $\phi_{k}^{(s)}(x)$ for $x \in \mathbb{R}$ and $k \in \mathbb{Z}$ such that $\left\{\phi_{k}^{(s)}\right\}_{k \in \mathbb{Z}}$ is a complete, orthogonal system for any valid $s$. Our method relies on the observation that the functions (1.1) are weighted maps of the canonical Fourier basis $e^{i k \theta}$ for $\theta \in[-\pi, \pi]$ (see e.g. [8], 25]). We first generalize the Fourier basis on $[-\pi, \pi]$ to have the properties we desire on the finite interval; we will then map the generalized Fourier basis to the real line and weight it accordingly to achieve the desired rate of decay.

2.1. Notation and setup. We reserve the variables $x, z, \theta$, and $r$ as independent variables on certain domains and list the domains and transformations in Table 1 . The domain $\mathbb{T}^{+}$is the upper-half of the unit circle in the complex plane. We will occasionally use the notation e.g. $r(\theta)$, and the relationship between these variables will then be dictated by Table 1. The domains of definition for $\theta, z$, and $x$ will be extended to $[-\pi, \pi], \mathbb{T}$, and $\mathbb{R}$, respectively.

Let us denote $L^{2}(A, B ; w)=L_{w}^{2}(A, B)$ the space of square integrable functions $f: A \rightarrow B$ under the weight $w$. We endow $L_{w}^{2}(A, B)$ with the conjugate bilinear inner product; the notation for this inner product is $\langle\cdot, \cdot\rangle_{w}$. The omission of $w$ indicates the unit weight measure. The norm on this space will be denoted $\|\cdot\|_{w}$. The following weight functions will be used:

$$
\begin{aligned}
& w_{r}^{(\alpha, \beta)}(r)=(1-r)^{\alpha}(1+r)^{\beta}, \\
& w_{\theta}^{(\gamma, \delta)}(\theta)=w_{r}^{(\delta, \gamma)}(r(\theta))=(1+\cos \theta)^{\gamma}(1-\cos \theta)^{\delta}, \\
& w_{x}^{(s, t)}(x)=w_{\theta}^{(s, t)}(\theta(x))=\frac{2^{s+t}}{\left(1+x^{2}\right)^{s}}\left(\frac{x^{2 t}}{\left(1+x^{2}\right)^{t}}\right) .
\end{aligned}
$$

In addition, we will make use of phase-shifted square roots of $w_{x}^{(s, t)}$ and $w_{\theta}^{(\gamma, \delta)}$, which we define as:

$$
\sqrt[*]{w_{x}^{(s, t)}(x)}:=\sqrt{w_{x}^{(s, t)}} \exp \left[\frac{i(s+t)}{2}(\pi-\theta(x))\right]=\frac{2^{\left(\frac{s+t}{2}\right)} x^{t}}{(x-i)^{s+t}}
$$




$$
\begin{aligned}
\sqrt[*]{w_{\theta}^{(\gamma, \delta)}(\theta)} & =\sqrt[*]{w_{x}^{(\gamma, \delta)}(x(\theta))} \\
& =2^{\left(\frac{\gamma+\delta}{2}\right)} \sin ^{\delta}\left(\frac{\theta}{2}\right) \cos ^{\gamma}\left(\frac{\theta}{2}\right) \exp \left[\frac{i(\gamma+\delta)}{2}(\pi-\theta)\right] .
\end{aligned}
$$

2.2. Jacobi polynomials. The classical Jacobi polynomials $P_{n}^{(\alpha, \beta)}$ are a family of orthogonal polynomials 24] that have been used extensively in many applications. They are defined through the Jacobi differential equation

$$
\left(1-r^{2}\right) \rho^{\prime \prime}+[\beta-\alpha-(\alpha+\beta+2) r] \rho^{\prime}+n(n+\alpha+\beta+1) \rho=0, \quad r \in(-1,1) .
$$

For $\alpha, \beta>-1, n \in \mathbb{N}_{0}$ the only polynomial solution $\rho=P_{n}^{(\alpha, \beta)}(r)$ is a polynomial of degree $n$. The restriction $\alpha, \beta>-1$ is necessary to ensure integrability of the weight and existence of an $L^{2}$-constant function solution. The family of polynomials $\left\{P_{n}^{(\alpha, \beta)}(x)\right\}_{n=0}^{\infty}$ is complete and orthogonal in $L^{2}\left((-1,1), \mathbb{R} ; w_{r}^{(\alpha, \beta)}\right)$. We denote the normalized polynomials as

$$
\tilde{P}_{n}^{(\alpha, \beta)}(r)=\frac{P_{n}^{(\alpha, \beta)}(r)}{\left\|P_{n}^{(\alpha, \beta)}\right\|_{w^{(\alpha, \beta)}}} .
$$

We require a minor generalization of Jacobi polynomials: performing a change of the dependent variable in (2.3), we obtain:

Lemma 2.1 (Jacobi Functions). The Jacobi functions defined as

$$
P_{n}^{(\alpha, \beta, a, b)}(r)=(1-r)^{a}(1+r)^{b} P_{n}^{(\alpha, \beta)}(r)
$$

satisfy the following properties:

(1) $\left\{P_{n}^{(\alpha, \beta, a, b)}(r)\right\}_{n \in \mathbb{N}_{0}}$ are orthogonal and complete in

$$
L^{2}\left((-1,1), \mathbb{R} ; w_{r}^{(\alpha-2 a, \beta-2 b)}\right) .
$$

(2) The $P_{n}^{(\alpha, \beta, a, b)}(r)$ are eigenfunctions $\rho_{n}(r)$ of the Sturm-Liouville problem

$$
-\frac{\mathrm{d}}{\mathrm{d} r}\left[p(r) \rho^{\prime}(r)\right]+q(r) \rho(r)-\lambda_{n} w(r) \rho(r)=0,
$$

with eigenvalues

$$
\lambda_{n}=n(n+\alpha+\beta+1)-2 a b+a(\beta+1)+b(\alpha+1) .
$$

As with the polynomials, we denote $\tilde{P}_{n}^{(\alpha, \beta, a, b)}$ as $L_{w}^{2}$-normalized versions of the Jacobi functions. We shall only require the result of Lemma 2.1 for $a=b=\frac{1}{2}$. Many of the results in this paper require the use of numerous well-known recurrence relations involving Jacobi polynomials; for a survey of these we refer to [1].

Finally, we present two classical notational conventions that we will use in the next section. The classical Jacobi polynomials for the cases $\alpha=\beta=-\frac{1}{2}$ and $\alpha=$ $\beta=+\frac{1}{2}$ are the Chebyshev polynomials of the first and second kinds, respectively. Recalling the relation $r=\cos \theta$, these polynomials are denoted $T_{n}(r)$ and $U_{n}(r)$ and they have the following concise representation as trigonometric polynomials:

$$
\begin{aligned}
\sqrt{\frac{\pi}{2}} \tilde{P}_{n}^{(-1 / 2,-1 / 2)}(r) & =T_{n}(r)=\cos (n \theta)=\cos [n \arccos (r)] \\
\sqrt{\frac{\pi}{2}} \tilde{P}_{n}^{(1 / 2,1 / 2)}(r) & =U_{n}(r)=\frac{\sin [(n+1) \theta]}{\sin \theta}=\frac{\sin [(n+1) \arccos (r)]}{\sin [\arccos (r)]} .
\end{aligned}
$$


2.3. Generalizing the Fourier basis. In this section we will generalize the canonical Fourier basis given by

$$
\Psi_{k}(\theta)=e^{i k \theta}
$$

Our methodology is based upon the following dissection of the Fourier basis for $k \neq 0$ :

$$
\begin{array}{rlccc}
e^{i k \theta} & = & \cos (k \theta) & + & i \sin (k \theta) \\
& = & \cos (|k| \theta) & + & i \operatorname{sgn}(k) \sin (|k| \theta) \\
& = & T_{|k|}(\cos \theta) & + & i \operatorname{sgn}(k) \sin (\theta) U_{|k|-1}(\cos \theta) \\
& = & \sqrt{\frac{\pi}{2}}[\underbrace{\tilde{P}_{|k|}^{(-1 / 2,-1 / 2)}(\cos \theta)}_{(a)}+ & & i \operatorname{sgn}(k) \underbrace{\sin (\theta) \tilde{P}_{|k|-1}^{(1 / 2)}(\cos \theta)}_{(b)}] .
\end{array}
$$

This breakdown suggests that we can construct more general Fourier-type functions by augmenting the type of polynomials employed.

The separation into terms $(a)$ and $(b)$ above elucidates the biorthogonal decomposition of the Fourier basis. In this case, the biorthogonality is manifested as an even-odd separation. Suppose we wish to generate a basis set orthogonal under the weight $1+\cos \theta=1+r$. Naturally, we can do this for basis $(a)$ by changing the second Jacobi class parameter from $\beta=-\frac{1}{2}$ to $\beta=+\frac{1}{2}$. In order to do this for basis (b), we use Lemma 2.1 .

Both basis sets $\tilde{P}_{n}^{(\alpha, \beta)}$ and $\tilde{P}_{n}^{(\alpha+1, \beta+1,1 / 2,1 / 2)}$ are orthogonal under the weight $w_{r}^{(\alpha, \beta)}$. For example, if we set $\alpha=\beta=-\frac{1}{2}$ and add these two basis sets together with the appropriate scaling factors, then we exactly recover the Fourier basis by reversing the dissection steps above. This technique has already been used by Szegö 24 to determine orthogonal polynomials on the unit circle. Because the statement in [24] is merely a passing comment and is a markedly different result than what we desire, we present the following theorem:

Theorem 2.2 (cf. Szegö 24], equation (11.5.4)). For any $\gamma>-\frac{1}{2}$, the functions

$$
\begin{aligned}
& \Psi_{k}^{(\gamma)}(\theta) \\
& \quad= \begin{cases}\frac{1}{\sqrt{2}} \tilde{P}_{0}^{(-1 / 2, \gamma-1 / 2)}(\cos \theta), & k=0, \\
\frac{1}{2}\left[\tilde{P}_{|k|}^{(-1 / 2, \gamma-1 / 2)}(\cos \theta)+i \operatorname{sgn}(k) \sin (\theta) \tilde{P}_{|k|-1}^{(1 / 2, \gamma+1 / 2)}(\cos \theta)\right], & k \neq 0\end{cases}
\end{aligned}
$$

are complete and orthonormal in $L^{2}\left((-\pi, \pi), \mathbb{C} ; w_{\theta}^{(\gamma, 0)}\right)$.

Proof. For orthonormality, it suffices to show:

(1) $\left\langle\tilde{P}_{|k|}^{(-1 / 2, \gamma-1 / 2)}(\cos \theta), \tilde{P}_{|l|}^{(-1 / 2, \gamma-1 / 2)}(\cos \theta)\right\rangle_{w_{\theta}^{(\gamma, 0)}}=2 \delta_{|k|,|l|}$.

(2) $\left\langle\sin \theta \tilde{P}_{|k|-1}^{(1 / 2, \gamma+1 / 2)}(\cos \theta), \sin \theta \tilde{P}_{|l|-1}^{(1 / 2, \gamma+1 / 2)}(\cos \theta)\right\rangle_{w_{\theta}^{(\gamma, 0)}}=2 \delta_{|k|,|l|}$, for $k, l \neq 0$.

(3) $\left\langle\tilde{P}_{|k|}^{(-1 / 2, \gamma-1 / 2)}(\cos \theta), \sin \theta \tilde{P}_{|l|-1}^{(1 / 2, \gamma+1 / 2)}(\cos \theta)\right\rangle_{w_{\theta}^{(\gamma, 0)}}=0$, for $l \neq 0$.

These three properties follow from using the orthogonality relations of Jacobi polynomials and Lemma 2.1.

For completeness we note that any function $f \in L^{2}$ can be decomposed into an even $f_{e}$ and an odd $f_{o}$ part. That $f_{e}$ is representable is clear from the fact that 
$\tilde{P}_{n}^{(-1 / 2, \gamma-1 / 2)}(\cos \theta)$ is complete over even functions on $\theta \in[-\pi, \pi]$. Similarly, the collection of functions $\sin \theta \tilde{P}_{n}^{(-1 / 2, \gamma-1 / 2)}$ is complete over all $L^{2}$-odd functions $f_{o}$. Linearity and orthogonality of the even and odd parts yield the result.

Remark 2.3. Szegö 24 gives a more general result that involves orthogonality over the weight $w_{\theta}^{(\gamma, \delta)}$ for $\delta \neq 0$. We do not require this level of generality. It is possible to generalize Szegö's result: he derived polynomials on the unit circle orthogonal with respect to $w_{\theta}^{(\gamma, \delta)}$. By using Lemma 2.1 with $a, b$ different from $\frac{1}{2}$, we can derive non-polynomial basis sets that are orthogonal under a great variety of weights. These functions naturally may not be smooth on $\theta \in[-\pi, \pi]$ if the quantity $(1-r)^{a}(1+r)^{b}$ cannot be smoothly extended in periodic $\theta$-space.

We will refer to the functions (2.4) as either the generalized Fourier series, or the Szegö-Fourier functions. We can also distribute the weight function onto the basis functions, which yields orthogonality in the unweighted $L^{2}$-norm:

Corollary 2.4. For any $\gamma>-\frac{1}{2}$, the functions

$$
\begin{aligned}
& \psi_{k}^{(\gamma)}(\theta)= \\
& \begin{cases}\frac{\sqrt[*]{w_{\theta}^{(\gamma, 0)}}}{\sqrt{2}} \tilde{P}_{0}^{(-1 / 2, \gamma-1 / 2)}(\cos \theta), & k=0, \\
\frac{\sqrt[*]{w_{\theta}^{(\gamma, 0)}}}{2}\left[\tilde{P}_{|k|}^{(-1 / 2, \gamma-1 / 2)}(\cos \theta)+i \operatorname{sgn}(k) \sin (\theta) \tilde{P}_{|k|-1}^{(1 / 2, \gamma+1 / 2)}(\cos \theta)\right], & k \neq 0\end{cases}
\end{aligned}
$$

are complete and orthonormal in $L^{2}((-\pi, \pi), \mathbb{C})$.

Due to the properties of $\sqrt[*]{w_{\theta}^{(\gamma, 0)}}$ given in (2.2), the functions $\psi_{k}^{(\gamma)}(\theta)$ decay like $\left(\cos \frac{\theta}{2}\right)^{\gamma}$ at $\theta= \pm \pi$. This is exemplified in Figure 1 where we plot the real and imaginary parts of the functions for $\gamma=2$. The even/odd behavior in $\theta$ for real/imaginary components depicted in Figure 1 depends on the even/odd parity of $\gamma$. (There is no such characterization possible when $\gamma \notin \mathbb{N}_{0}$.) Clearly for $\gamma=0$ we have $\Psi_{k}^{(0)}=\psi_{k}^{(0)}=\frac{1}{\sqrt{2 \pi}} e^{i k \theta}$, the canonical Fourier basis.

2.4. Mapping to the real line. Having developed the necessary preliminaries on the finite interval, we now jump to the infinite line $x \in \mathbb{R}$ using the mappings introduced in Table 1. We rewrite and relabel the functions $\Psi_{k}^{(\gamma)}(\theta)$ :

$$
\begin{aligned}
\Phi_{k}^{(s)}(x) & :=\Psi_{k}^{(s-1)}(\theta(x)) \\
& = \begin{cases}\frac{1}{\sqrt{2}} \tilde{P}_{0}^{(-1 / 2, s-3 / 2)}\left(\frac{1-x^{2}}{1+x^{2}}\right), & k=0, \\
\frac{1}{2}\left[\tilde{P}_{|k|}^{(-1 / 2, s-3 / 2)}\left(\frac{1-x^{2}}{1+x^{2}}\right)+\frac{2 i x \operatorname{sgn}(k)}{x^{2}+1} \tilde{P}_{|k|-1}^{(1 / 2, s-1 / 2)}\left(\frac{1-x^{2}}{1+x^{2}}\right)\right], & k \neq 0 .\end{cases}
\end{aligned}
$$

The above definition is valid for any $s>\frac{1}{2} . s=1$ corresponds to a mapping of the canonical Fourier basis (i.e., $s \doteq \gamma+1$ ). These functions are orthogonal over the weight $w_{x}^{(s, 0)}$. Remark 2.3 applies here as well: it is possible to generate functions orthogonal with weight function $w_{x}^{(s, t)}$ for $t>-\frac{1}{2}$, if desired. 


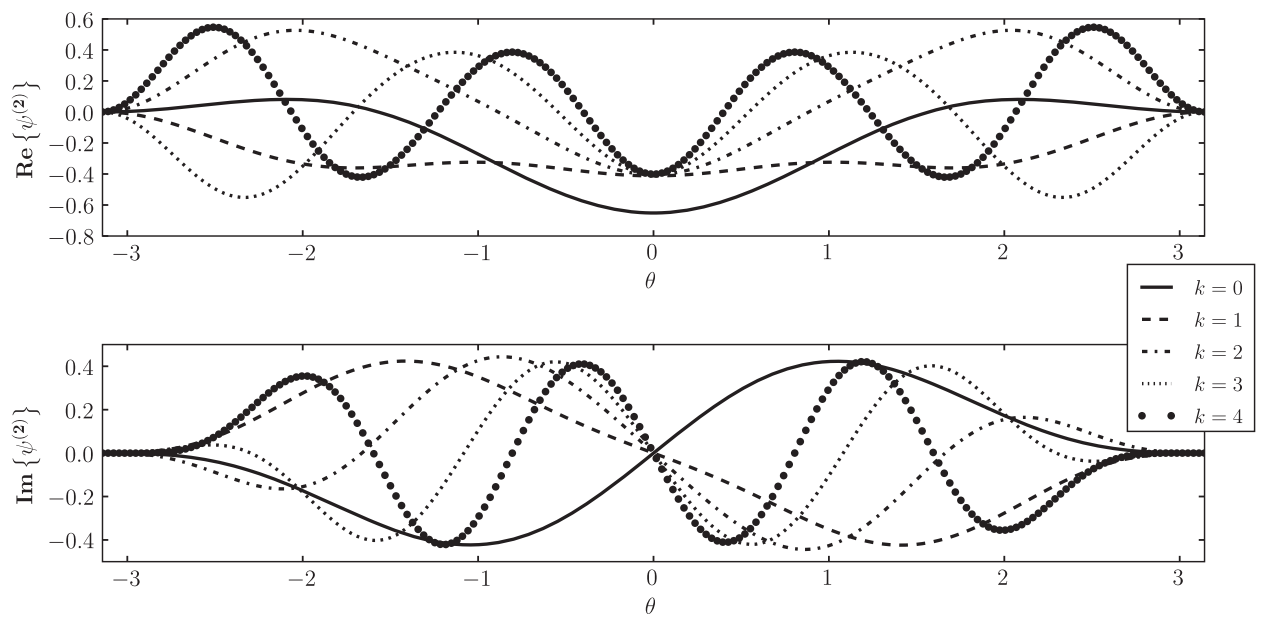

Figure 1. Plots of the weighted Szegö-Fourier functions $\psi_{k}^{(2)}(\theta)$ for $k=0,1,2,3$, and 4 . Real part (top) and imaginary part (bottom).

By following the route from Corollary 2.4 we can distribute the weight over the basis functions, and we choose the phase-shifted square root given in (2.1):

$$
\begin{aligned}
\phi_{k}^{(s)} & :=\sqrt[*]{w_{x}^{(s, 0)}} \Phi_{k}^{(s)}(x) \\
& = \begin{cases}\frac{2\left(\frac{s-1}{2}\right)}{(x-i)^{s}} \tilde{P}_{0}^{(-1 / 2, s-3 / 2)}\left(\frac{1-x^{2}}{1+x^{2}}\right), & k=0, \\
\frac{2^{\left(\frac{s}{2}-1\right)}}{(x-i)^{s}}\left[\tilde{P}_{|k|}^{(-1 / 2, s-3 / 2)}\left(\frac{1-x^{2}}{1+x^{2}}\right)+\frac{2 i x \operatorname{sgn}(k)}{x^{2}+1} \tilde{P}_{|k|-1}^{(1 / 2, s-1 / 2)}\left(\frac{1-x^{2}}{1+x^{2}}\right)\right], & k \neq 0 .\end{cases}
\end{aligned}
$$

The functions (2.5) are what we refer to as the generalized Wiener rational functions. At present our choice of weight function $\sqrt[*]{w_{x}^{(s, 0)}}$ instead of the usual square root $\sqrt{w_{x}^{(s, 0)}}$ to distribute the weight is mysterious. However, the corollary following the coming proposition provides part of the motivation.

Proposition 2.5. For any $s>\frac{1}{2}$, the functions $\Phi_{k}^{(s)}(x)$ are complete and orthonormal in $L^{2}\left(\mathbb{R}, \mathbb{C} ; w_{x}^{(s, 0)}\right)$. The functions $\phi_{k}^{(s)}(x)$ are complete and orthonormal in $L^{2}(\mathbb{R}, \mathbb{C})$. Furthermore, the decay rate of these functions can be characterized as

$$
\lim _{|x| \rightarrow \infty}\left|x^{t} \phi_{k}^{(s)}(x)\right|<\infty, \quad t \leq s .
$$

Corollary 2.6. Recalling the definition of Wiener's original basis functions $\phi_{n}(x)$ in (1.1), the following relation holds:

$$
\phi_{-n}^{(1)}(x) \equiv i \phi_{n}(x), \quad n \in \mathbb{N}_{0} .
$$




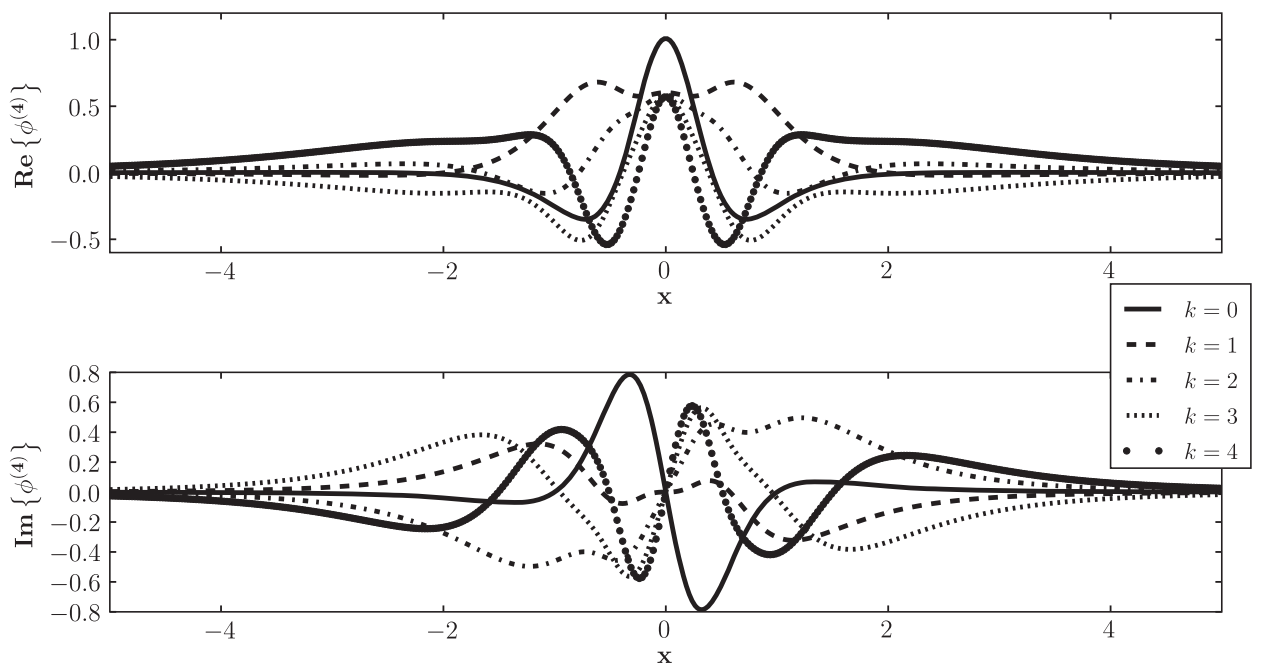

Figure 2. Plots of the functions $\phi_{k}^{(4)}(x)$ for $k=0,1,2,3,4$.

We show plots of the functions $\phi_{k}^{(4)}$ in Figure 2. The corollary is proven using the identity

$$
\sqrt{\frac{\pi}{2}} \tilde{P}_{n}^{(-1 / 2,-1 / 2)}(r(\theta))+i \sin \theta \sqrt{\frac{\pi}{2}} \tilde{P}_{n-1}^{(1 / 2,1 / 2)}(r(\theta))=e^{i n \theta}=\left(\frac{i-x}{i+x}\right)^{n}, \quad n>0 .
$$

We have thus shown that the orthogonal functions $\phi_{k}^{(s)}$ over the real line are indeed a generalization of Wiener's original basis set (modulo re-indexing). Furthermore, $\phi_{k}^{(s)}$ decays like $x^{-s}$ while retaining orthogonality under the unit weight measure. When $s$ is an integer, the functions are also purely rational: they are the division of one complex-valued polynomial in $x$ by another.

A more functional reason (compared to Corollary 2.6) to use the phase-shifted square root is that it can be written in the following convenient form:

$$
\sqrt[*]{w_{x}^{(s, 0)}}=\left[\frac{i}{\sqrt{2}}\left(1+e^{-i \theta(x)}\right)\right]^{s}
$$

The utility of this expression will become clear when we consider the connection problems in Section 3 ,

We have accomplished our goal of deriving basis functions satisfying tunable decay rate while maintaining $L^{2}$-orthogonality. However, it is not clear that these are useful functions. We will now present some properties of the basis and make the argument that these basis functions indeed are very useful for solving a variety of problems in scientific computing.

\section{BASIS PROPERTIES}

The generalized Wiener functions are composed of Jacobi polynomials, and so it is reasonable to expect that we can use the properties of the Jacobi polynomials to perform certain tasks using the Wiener basis. Indeed, we can form recurrence relations, connection coefficients, a Gauss-like quadrature, and obtain a sparsity result for the Galerkin stiffness matrix. 
3.1. Recurrence relations. Due to the strong dependence of the Szegö-Fourier functions on the Jacobi polynomials, they inherit five-term recurrence relations from the three-term recurrences for classical orthogonal polynomials 24]:

$$
\begin{aligned}
D_{n}^{(\gamma)} \Psi_{n+1}^{(\gamma)}=[ & \left.A_{n}^{(\gamma)} e^{i \theta}-B_{n}^{(\gamma)}\right] \Psi_{n}^{(\gamma)}+\left[A_{-n}^{(\gamma)} e^{-i \theta}-B_{-n}^{(\gamma)}\right] \Psi_{-n}^{(\gamma)} \\
& +C_{n}^{(\gamma)} \Psi_{n-1}^{(\gamma)}+C_{-n}^{(\gamma)} \Psi_{-(n-1)}^{(\gamma)},
\end{aligned}
$$

We give formulae for all the real-valued constants $A, B, C, D$ in Appendix $\mathrm{A}$ Note that since the $\Psi_{k}^{(\gamma)}$ are not polynomials in $z=e^{i \theta}$, there is not a three-term recurrence as there would normally be for orthogonal polynomials on the unit disk (unless of course $\gamma=0$ ). Although the above formulae are complex-valued fiveterm recurrence relations, they are no more difficult computationally than the pair of three-term recurrences necessary to generate $\tilde{P}_{n}^{(\alpha, \beta)}$ and $\tilde{P}_{n}^{(\alpha+1, \beta+1)}$ because $\Psi_{n}^{(\gamma)}$ is the complex conjugate of $\Psi_{-n}^{(\gamma)}$ and therefore does not need to be generated independently. In other words, direct use of any of the above five-term recurrences for generating the $\Psi_{k}^{(\gamma)}$ is just as expensive as forming $\Psi_{k}^{(\gamma)}$ by the even/odd synthesis of $\tilde{P}_{n}^{(\alpha, \beta)}$ and $\tilde{P}_{n}^{(\alpha+1, \beta+1)}$ in Theorem 2.2 .

Naturally, a recurrence relation for the unweighted $\Psi_{k}^{(\gamma)}(\theta)$ translates directly into one for the unweighted Wiener rational functions $\Phi_{k}^{(s)}(x)$. The weighted functions $\psi_{k}^{(\gamma)}(\theta)$ and $\phi_{k}^{(s)}(x)$ can be generated by first generating the unweighted functions and then multiplying by the phase-shifted square root $\sqrt[*]{w}$.

3.2. Connection problems. One advantage in using the generalized Wiener rational function basis is the ability to choose the parameter $s$, which reflects the rate of decay. In many applications, it may be useful to augment the basis functions mid-computation to suit the dynamics occurring at a particular time. In this case, one would like to be able to transfer from one basis to another while keeping the (finite-term) function expansion identical. We will see in Part II that this problem also appears in an algorithm utilizing the FFT to evaluate the expansion. In classical orthogonal polynomial theory, the problem of equating one expansion to another is known as the connection problem.

There are two main tasks on the infinite interval that require connections of some form:

(1) Usage of the fast Fourier transform - transforming $N$ nodal evaluations into $N$ modal coefficients (or vice-versa) for an expansion in $\phi^{(s)}$.

(2) For a given expansion in $\phi^{(s)}$ (i.e. a set of modal coefficients), translating this into a modal coefficient expansion in $\phi^{(S)}$ for some $s \neq S$.

In Part II where we outline computational considerations, we will address the above tasks in more detail. However, for now it suffices to note that these two tasks can be reduced to the following three connection problems in $\theta$-space:

(1) The $\Psi^{(\gamma)}-\Psi^{(\Gamma)}$ connection (a necessary ingredient for all connection-like tasks).

(2) The $\Psi^{(\gamma)}-\psi^{(\gamma)}$ connection (a generalization of the FFT task).

(3) The $\psi^{(\gamma)}-\psi^{(\Gamma)}$ connection (identical to modification of $s$ ).

Modification of any of the following finite-interval algorithms for the infinite interval is trivial: the relations $\Psi_{k}^{(\gamma)}(\theta) \equiv \Phi_{k}^{(s)}(x), \psi_{k}^{(\gamma)}(\theta) \equiv \phi_{k}^{(s)}(x)$, and $\gamma:=s-1$ allow 
for us to easily employ the same operations, whether we want to do it in $\theta$-space or $x$-space.

3.2.1. The $\Psi-\Psi$ connection problem. Suppose we have a function

$$
f \in L^{2}\left((-\pi, \pi), \mathbb{C} ; w_{\theta}^{(\gamma, 0)}\right) \bigcap L^{2}\left((-\pi, \pi), \mathbb{C} ; w_{\theta}^{(\Gamma, 0)}\right)
$$

with a Fourier expansion for some $\gamma>-\frac{1}{2}$ :

$$
f(x)=\sum_{k \in \mathbb{Z}} \hat{f}_{k}^{(\gamma)} \Psi_{k}^{(\gamma)}=\sum_{k \in \mathbb{Z}} \hat{f}_{k}^{(\Gamma)} \Psi_{k}^{(\Gamma)} .
$$

We assume that the $\gamma$ coefficients are known and we seek the $\Gamma$ coefficients. The shift $\Gamma-\gamma$ can take values in the interval $\left(-\frac{1}{2}-\gamma, \infty\right)$. Define the connection coefficients as

$$
\lambda_{k, l}^{\Psi}=\left\langle\Psi_{l}^{(\gamma)}, \Psi_{k}^{(\Gamma)}\right\rangle_{w_{\theta}^{(\Gamma, 0)}},
$$

where we have suppressed the dependence of $\lambda$ on $\gamma$ and $\Gamma$. The connection problem is solved via the relation

$$
\hat{f}_{k}^{(\Gamma)}=\sum_{\substack{l \in \mathbb{Z},|l| \geq|k|}} \hat{f}_{l}^{(\gamma)} \lambda_{k, l}^{\Psi} .
$$

We will show that, for integer values of the shift $\Gamma-\gamma$, the connection problem can be solved inexpensively. To be precise, we will show that for $G \in \mathbb{N}$, (3.1) reduces to

$$
\hat{f}_{k}^{(\gamma+G)}=\sum_{k+G \geq|l| \geq|k|} \hat{f}_{l}^{(\gamma)} \lambda_{k, l}^{\Psi}
$$

That is, only $2(G+1)$ operations per coefficient are necessary to solve the connection problem (independent of $k$, and of any truncation size $N$ ). We refer to the above collapse of the infinite connection problem (3.1) into the finite $N$-independent problem (3.2) as a sparse connection.

In order to relate one Fourier function to another, we first recall a result from 21] using Jacobi polynomial recurrences that state that the connection coefficients binding one Jacobi polynomial class to another are sparse in certain circumstances.

Lemma 3.1. For any $\alpha, \beta>-1$ and any $A, B, \in \mathbb{N}_{0}$, the connection problem

$$
f(r)=\sum_{n=0}^{\infty} \hat{f}_{n}^{(\alpha, \beta)} \tilde{P}_{n}^{(\alpha, \beta)}(r) \longrightarrow f(r)=\sum_{n=0}^{\infty} \hat{f}_{n}^{(\alpha+A, \beta+B)} \tilde{P}_{n}^{(\alpha+A, \beta+B)}(r),
$$

can be solved exactly via the relation

$$
\hat{f}_{n}^{(\alpha+A, \beta+B)}=\sum_{m=0}^{A+B} \lambda_{n, n+m}^{P} \hat{f}_{n+m}^{(\alpha, \beta)}
$$

In the above we have suppressed the dependence of $\lambda^{P}$ on $\alpha, \beta, A$,and $B$, but in the sequel we shall occasionally refer to the above coefficients as $\lambda_{n ; m}^{P ;(\alpha, \beta, A, B)}$.

To obtain the Jacobi-Jacobi connection coefficients $\lambda^{P}$, one may use explicit formulae given in [19] or [2], or one may utilize the algorithm given in 21].

The above result can be expanded to apply to the Szegö-Fourier functions $\Psi_{k}^{(\gamma)}(\theta)$ and the corresponding mapped functions $\Phi_{k}^{(s)}(x)$. 
Proposition 3.2. For any $\gamma>-\frac{1}{2}$ and any $G \in \mathbb{N}$, the connection problem

$$
f(\theta)=\sum_{k=-\infty}^{\infty} \hat{f}_{k}^{(\gamma)} \Psi_{k}^{(\gamma)}(\theta) \longrightarrow f(\theta)=\sum_{k=-\infty}^{\infty} \hat{f}_{k}^{(\gamma+G)} \Psi_{k}^{(\gamma+G)}(\theta),
$$

can be solved exactly via the relation

$$
\hat{f}_{k}^{(\gamma+G)}=\sum_{l=|k|}^{|k|+G} \lambda_{k, l}^{\Psi} \hat{f}_{l}^{(\gamma)}+\sum_{l=-|k|-G}^{-|k|} \lambda_{k, l}^{\Psi} \hat{f}_{l}^{(\gamma)} .
$$

Note that (3.4) is exactly of the form (3.2). By making the connection $s-1 \longleftrightarrow$ $\gamma$, we recover $\lambda_{k, l}^{\Phi} \equiv \lambda_{k . l}^{\Psi}$, where $\Phi_{k}^{(s)}(x)$ are the maps of the Szegö-Fourier functions $\Psi_{k}^{(\gamma)}$. This also yields the functional connection

$$
\Psi_{m}^{(\gamma)}(\theta)= \begin{cases}\sum_{|k| \leq m} \lambda_{k, m}^{\Psi} \Psi_{k}^{(\gamma+G)}(\theta), & |m| \leq G \\ \sum_{m-G \leq|k| \leq m} \lambda_{k, m}^{\Psi} \Psi_{k}^{(\gamma+G)}(\theta), & |m|>G,\end{cases}
$$

i.e., $\Psi_{m}^{(\gamma)}$ is a linear combination of at most $2 G+1$ functions $\Psi_{k}^{(\gamma+G)}$. The Fourier recurrence relation (3.5) mirrors (3.4) in a way similar to the parallels between known Jacobi polynomial recurrences.

We now illustrate how to calculate the Szegö-Fourier connection coefficients $\lambda^{\Psi}$ in Proposition 3.2 from the Jacobi coefficients $\lambda^{P}$. In the following, we make use of the notation:

$$
n:=|k|-1, \quad \alpha=-\frac{1}{2}, \quad \beta=\gamma-\frac{1}{2} .
$$

From the definition of $\Psi_{k}^{(\gamma)}$ in (2.4) we have

$$
\begin{aligned}
\tilde{P}_{n+1}^{(\alpha, \beta)} & =\Psi_{k}^{(\gamma)}+\Psi_{-k}^{(\gamma)} \\
& =\Psi_{|k|}^{(\gamma)}-\Psi_{-|k|}^{(\gamma)} \\
& \tilde{P}_{0}^{(\alpha, \beta)}=\sqrt{2} \Psi_{0}^{(\gamma)} .
\end{aligned} \geq 0
$$

Therefore, from the modes $\hat{f}_{k}^{(\gamma)}$ we can derive two sets of Jacobi modes:

$$
\begin{array}{rlrl}
\hat{e}_{n}^{(\alpha, \beta)} & =\hat{f}_{n}^{(\gamma)}+\hat{f}_{-n}^{(\gamma)}, & & n \geq 1, \\
\hat{o}_{n}^{(\alpha+1, \beta+1)} & =\hat{f}_{n+1}^{(\gamma)}-\hat{f}_{-n-1}^{(\gamma)}, & & n \geq 0, \\
\hat{e}_{0}^{(\alpha, \beta)} & =\sqrt{2} \hat{f}_{0}^{(\gamma)} . &
\end{array}
$$

The Jacobi modes $\hat{e}_{n}$ are modes in an expansion in polynomials $\tilde{P}_{n}^{(\alpha, \beta)}$ and the modes $\hat{o}_{n}$ are for an expansion in $\tilde{P}_{n}^{(\alpha+1, \beta+1)}$. With these modes in hand, we can use the Jacobi connection coefficients to promote the coefficients using Lemma 3.1 when $n \geq 0$ :

$$
\begin{aligned}
\hat{e}_{n}^{(\alpha, \beta+G)} & =\sum_{m=0}^{G} \lambda_{n, n+m}^{P} \hat{e}_{n+m}^{(\alpha, \beta)}, & & \text { where } \lambda^{P}=\lambda^{P(\alpha, \beta, 0, G)}, \\
\hat{o}_{n}^{(\alpha+1, \beta+G+1)} & =\sum_{m=0}^{G} \lambda_{n, n+m}^{P} \hat{o}_{n+m}^{(\alpha+1, \beta+1)}, & & \text { where } \lambda^{P}=\lambda^{P(\alpha+1, \beta+1,0, G) .} .
\end{aligned}
$$

Finally, we redistribute the modes back into Szegö-Fourier form to obtain:

$$
\begin{aligned}
& \hat{f}_{n}^{(\gamma+G)}=\frac{1}{2}\left[\hat{e}_{n}^{(\alpha, \beta+G)}+\hat{o}_{n-1}^{(\alpha+1, \beta+1+G)}\right], \quad n \geq 1, \\
& \hat{f}_{-n}^{(\gamma+G)}=\frac{1}{2}\left[\hat{e}_{n}^{(\alpha, \beta+G)}-\hat{o}_{n-1}^{(\alpha+1, \beta+1+G)}\right], \quad n \geq 1, \\
& \hat{f}_{0}^{(\gamma+G)}=\frac{\hat{e}_{0}^{(\alpha, \beta+G)}}{\sqrt{2}} \text {. }
\end{aligned}
$$




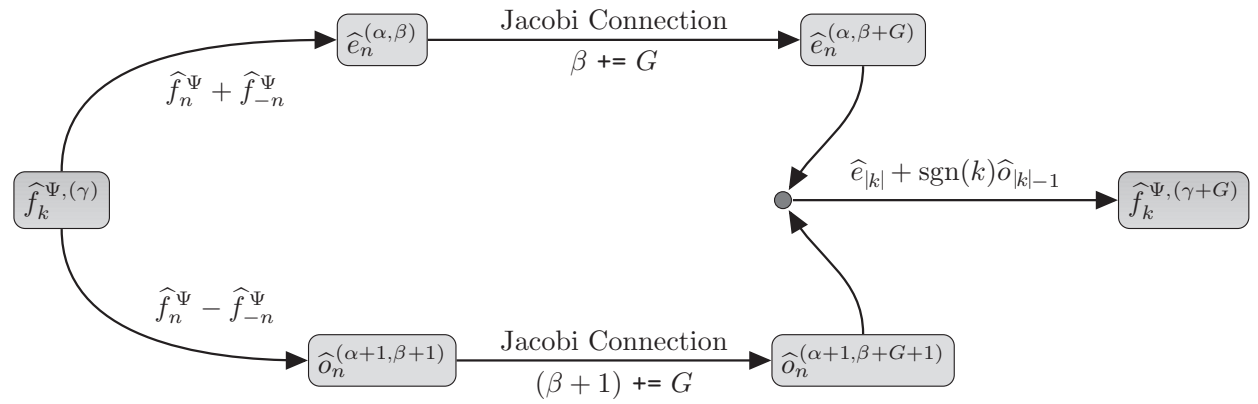

FiguRE 3. Illustration of steps taken to perform $\Psi-\Psi$ connections.

The operator $+=$ is the addition-assignment operator.

The whole procedure is illustrated graphically in Figure 3. The Szegö-Fourier connection coefficients in (3.2) are given explicitly as

$$
\lambda_{k, \pm(|k|+m)}^{\Psi}= \begin{cases}\frac{1}{2}\left[\lambda_{|k|,|k|+m}^{P ;(\alpha, \beta, 0, G)} \pm \operatorname{sgn}(k) \lambda_{|k|,|k|+m}^{P ;(\alpha+1, \beta+1,0, G)}\right], & |k| \geq 1, \\ \frac{1}{\sqrt{2}} \lambda_{0, m}^{P ;(\alpha, \beta, 0, G)}, & k=0 .\end{cases}
$$

The above equation is restricted to $0 \leq m \leq G$. This completes the $\Psi$ - $\Psi$ connection problem. The reverse connection problem (converting $\hat{f}_{k}^{\Psi,(\gamma+G)}$ modes to $\hat{f}_{k}^{\Psi,(\gamma)}$ modes) is solved by reversing the above procedure (all steps are invertible) and using the fact that the forward Jacobi connection problem with integer-valued parameter separation is banded upper-triangular and thus the backward connection is $\mathcal{O}(N)$ calculable sequentially via back-substitution. See [21.

We have determined how to quickly and exactly accomplish the connection problems for the unweighted functions

$$
\sum_{k} \hat{f}_{k}^{\Psi,(\gamma)} \Psi_{k}^{(\gamma)}(\theta) \longleftrightarrow \sum_{k} \hat{f}_{k}^{\Psi,(\gamma+G)} \Psi_{k}^{(\gamma+G)}(\theta),
$$

in $\mathcal{O}(N)$ time where $N$ is the total number of modes when $G \in \mathbb{Z}$. These connections can be performed by utilizing the connection coefficients in (3.6) along with the sparse connection result of Proposition 3.2. For $G \notin \mathbb{Z}$, there is no sparse connection result for the modes, and so while the connection coefficients $\lambda_{k, l}^{\Psi}$ can still be calculated based on known connection coefficients for Jacobi polynomials, the coefficients do not terminate finitely.

3.2.2. The $\Psi-\psi$ connection problem. Consider the following problem: let

$$
f \in L^{2}((-\pi, \pi), \mathbb{C}) .
$$

We assume $\gamma \geq 0$ and consider two expansions:

$$
f(\theta)=\sum_{k \in \mathbb{Z}} \hat{f}_{k}^{\Psi} \Psi_{k}^{(\gamma)}(\theta)=\sum_{k \in \mathbb{Z}} \hat{f}_{k}^{\psi} \psi_{k}^{(\gamma)}(\theta) .
$$

The modal coefficients are defined in the following way:

$$
\hat{f}_{k}^{\Psi,(\gamma)}=\left\langle f, \Psi_{k}^{(\gamma)}\right\rangle_{w_{\theta}^{(\gamma)}}, \quad \hat{f}_{k}^{\psi,(\gamma)}=\left\langle f, \psi_{k}^{(\gamma)}\right\rangle .
$$


We assume that the modal coefficients for the uppercase (unweighted function) expansion are known and that we wish to determine the lowercase modes $\hat{f}^{\psi}$. From the definitions of the modal coefficients, we can rewrite the lowercase modes as

$$
\hat{f}_{k}^{\psi,(\gamma)}=\left\langle f, \psi_{k}^{(\gamma)}\right\rangle=\left\langle f\left[\sqrt[*]{w_{\theta}^{(\gamma)}}\right]^{-\gamma}, \Psi_{k}^{(\gamma)}\right\rangle_{w_{\theta}^{(\gamma)}} .
$$

Now recall (2.6) and define

$$
g(\theta):=f\left[\sqrt[*]{w_{\theta}^{(\gamma)}}\right]^{-1}=f \times\left[\frac{\sqrt{2}}{i\left(1+e^{-i \theta}\right)}\right]^{\gamma} .
$$

Suppose that $\gamma=G \in \mathbb{N}_{0}$ and that we can somehow find the modal coefficients

$$
\hat{g}_{k}^{\Psi,(0)}=\left\langle g, \Psi_{k}^{(0)}\right\rangle \text {. }
$$

Then we can use the $\Psi-\Psi$ connection problem outlined in Section 3.2.1 to determine the modal coefficients $\hat{g}_{k}^{\Psi,(G)}$ due to the sparse connection. To find the modal coefficients $\hat{g}_{k}^{\Psi,(0)}$, assume that we have the modal coefficients $\hat{f}_{k}^{\Psi,(0)}$. Then (3.7) implies that

$$
\sum_{m=0}^{G}\left(\begin{array}{c}
G \\
m
\end{array}\right) \hat{g}_{k+m}^{\Psi,(0)}=\hat{f}_{k}^{\Psi,(0)}\left(\frac{\sqrt{2}}{i}\right)^{G} .
$$

If we assume a finite expansion so that $\hat{g}_{k}=0$ for $|k|>2 N+1$, then we can solve (3.8) via back-substitution. Note that determining each coefficient costs $\mathcal{O}(G)$ operations, independent of $N$; this is a similar operation count to the $\Psi-\Psi$ connection cost.

Finally, we must obtain $\hat{f}_{k}^{\Psi,(0)}$ from the given input $\hat{f}_{k}^{\Psi,(G)}$. However, this is another $\Psi-\Psi$ connection (albeit in reverse). Therefore, the three steps to take us from $\hat{f}_{k}^{\Psi,(G)}$ modes to $\hat{f}_{k}^{\psi,(G)}$ modes are

(1) Compute $\hat{f}_{k}^{\Psi,(0)}$ from $\hat{f}_{k}^{\Psi,(G)}$, as a (backward) $\Psi-\Psi$ connection.

(2) Compute $\hat{g}_{k}^{\Psi,(0)}$ from $\hat{f}_{k}^{\Psi,(0)}$ using (3.8).

(3) Compute $\hat{f}_{k}^{\psi,(G)} \equiv \hat{g}_{k}^{\Psi,(G)}$ from $\hat{g}_{k}^{\Psi,(0)}$, a (forward) $\Psi-\Psi$ connection.

This is illustrated in Figure 4. For an expansion with $N$ modes, all three steps have $\mathcal{O}(N G)$ cost asymptotically. The backward connection problem (determining $\hat{f}^{\Psi,(G)}$ from $\hat{f}^{\psi,(G)}$ ) is also computable in $\mathcal{O}(N G)$ operations, and is accomplished by reversing the above operations.

Note that if $\gamma \notin \mathbb{N}_{0}$, then all of these steps break down: the $\Psi$ - $\Psi$ connection is not sparse, and (3.8) is not valid since $\gamma$ is not an integer in (3.7).

This particular connection problem is not necessarily useful explicitly since in many of our applications we will have direct access to $\hat{f}^{\Psi,(0)}$, but each of the pieces necessary for this computation are used extensively both in modification of the decay parameter $s$ and application of the FFT.

3.2.3. Modification of $s$ : The $\psi-\psi$ connection. We have now developed the necessary tools for the modification of $s$, i.e., the $\psi-\psi$ connection problem. We assume that $G, F \in \mathbb{N}$ and that we know connection coefficients of some function $f \in L^{2}$ for an expansion in $\psi^{(F)}$, and wish to obtain the coefficients for a $\psi^{(G)}$ expansion. The whole procedure can be accomplished in three steps: 


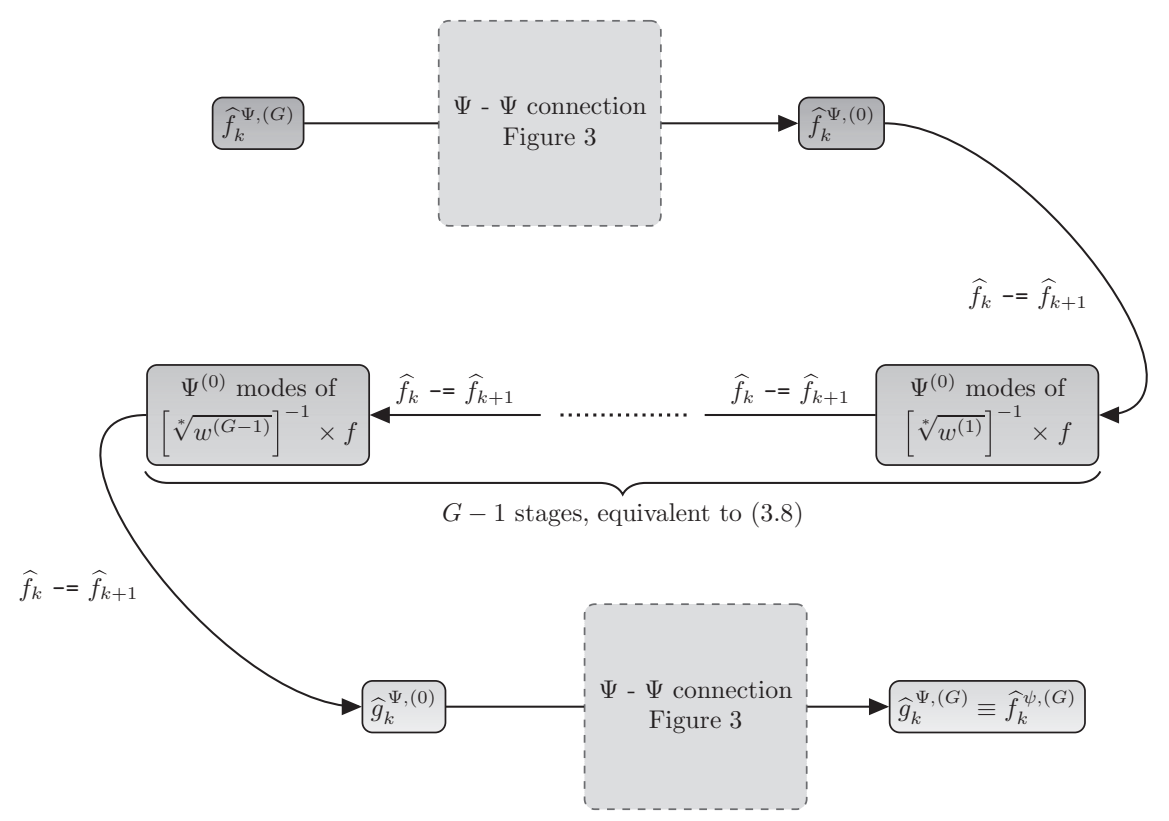

Figure 4. Flowchart representation of a $\Psi-\psi$ connection. The operator $-=$ is the subtraction-assignment operator.

(1) Obtain expansion coefficients for $f \times\left[\sqrt[*]{w_{\theta}^{(F)}}\right]^{-1}$ in $\Psi^{(0)}$ ( $\Psi-\psi$ connection).

(2) Obtain expansion coefficients for $f \times\left[\sqrt[*]{w_{\theta}^{(G)}}\right]^{-1}$ in $\Psi^{(0)}$ (Fourier connection).

(3) Obtain the sought expansion coefficients of $f$ in $\psi^{(G)}$ $(\Psi-\psi$ connection $)$.

Step 2 is easily performed using a version of (3.8) by noting the relation between $f \times\left[\sqrt[*]{w_{\theta}^{(G)}}\right]^{-1}$ and $f \times\left[\sqrt[*]{w_{\theta}^{(F)}}\right]^{-1}$ with knowledge of the canonical Fourier expansion coefficients $\left(\Psi^{(0)}(\theta)\right)$. This is shown in Figure 5 for the special case $F=3, G=5$.

Note that this particular connection problem is very amenable to an FFT+collocation approach whereas the algorithm we have laid out is a 'Galerkin' approach. The problem with the collocation approach is that it requires $\mathcal{O}(N \log N)$ operations with two FFTs, whereas the above algorithm requires only $\mathcal{O}(N G)$ steps.

3.3. Quadrature. We now turn to quadrature rules that will be used to compute integrals over the real line. We adopt the following notation: the pair $\left\{r_{n}^{(\alpha, \beta)}, \omega_{n}^{(\alpha, \beta)}\right\}_{n=1}^{N}$ denotes the $N$-point Gauss quadrature for the Jacobi polynomials of class $(\alpha, \beta)$. We suppress the dependence of $r_{n}^{(\alpha, \beta)}$ and $\omega_{n}^{(\alpha, \beta)}$ on $N$, and 


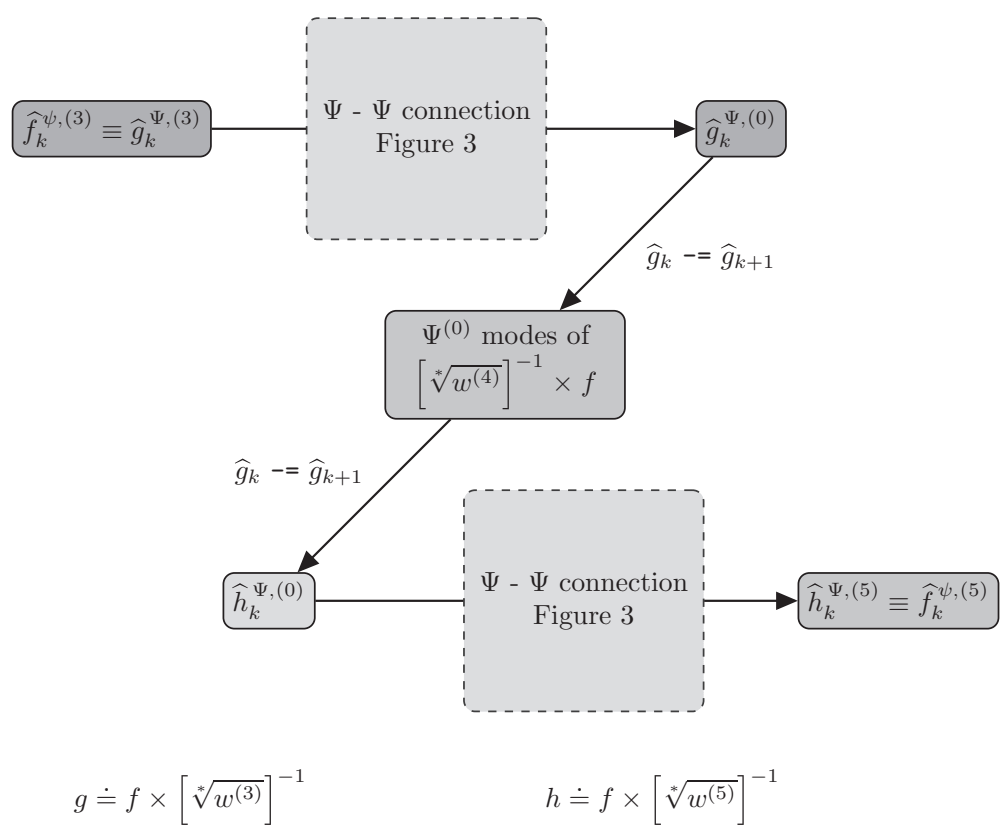

Figure 5. Flowchart of operations for modification of $s$. The operator $-=$ is the subtraction-assignment operator.

denote $\left\{r_{n}^{(\alpha, \beta) ; G R}, \omega_{n}^{(\alpha, \beta) ; G R}\right\}_{n=1}^{N}$ as the $N$-point Gauss-Radau quadrature with the fixed node $r_{N}^{(\alpha, \beta) ; \mathrm{GR}} \equiv 1$. We assume for clarity of presentation that the nodes are ordered by $n$, e.g., $r_{n-1}^{(\alpha, \beta)}<r_{n}^{(\alpha, \beta)}$.

To construct an $N$-point quadrature rule associated with the functions $\Psi_{k}^{(\gamma)}(\theta)$ when $N$ is even, define

$$
\theta_{n}^{(\gamma)}= \begin{cases}-\arccos \left(r_{n}^{(-1 / 2, \gamma-1 / 2)}\right), & 1 \leq n \leq \frac{N}{2}, \\ -\theta_{N+1-n}^{(\gamma)}, & \frac{N}{2}+1 \leq n \leq N,\end{cases}
$$

where $r_{n}^{(\alpha, \beta)}$ comes from an $\frac{N}{2}$-point quadrature rule, and

$$
\Omega_{n}^{(\gamma)}= \begin{cases}\omega_{n}^{(-1 / 2, \gamma-1 / 2)}, & 1 \leq n \leq \frac{N}{2} \\ \Omega_{N+1-n}^{(\gamma)}, & \frac{N}{2}+1 \leq n \leq N\end{cases}
$$

where $\omega_{n}^{(\alpha, \beta)}$ comes from an $\frac{N}{2}$-point quadrature rule.

If $N$ is odd, define

$$
\theta_{n}^{(\gamma)}= \begin{cases}-\arccos \left(r_{n}^{(-1 / 2, \gamma-1 / 2) ; \mathrm{GR}}\right), & 1 \leq n \leq \frac{N+1}{2} \\ \theta_{N+1-n}^{(\gamma)}, & \frac{N+3}{2} \leq n \leq N\end{cases}
$$




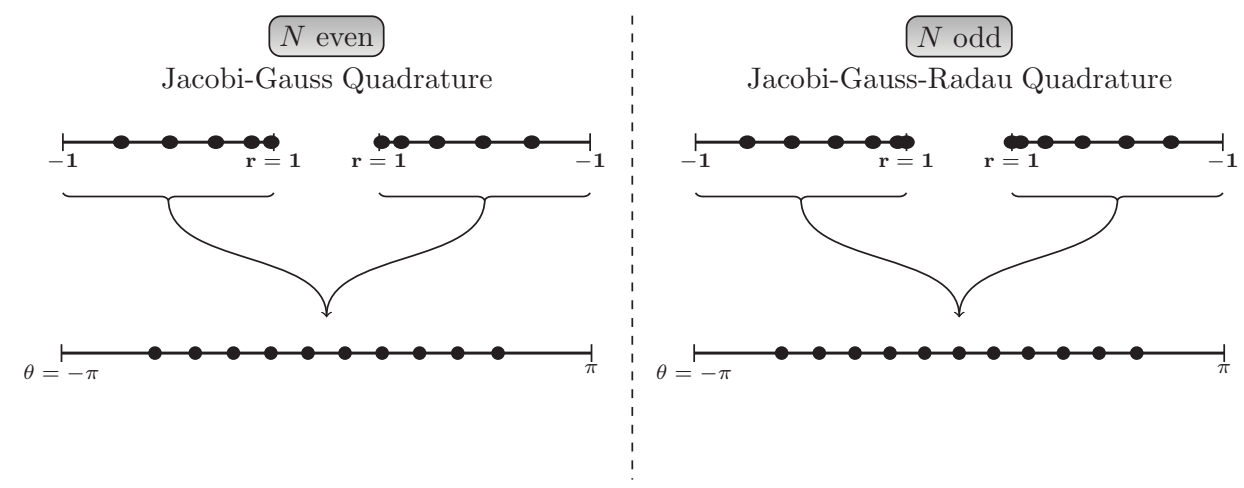

Figure 6. Construction of Gauss-type quadrature for generalized Fourier functions. The new quadrature rules are symmetric combinations of Jacobi-Gauss-type quadrature rules. The constructions shown are accurate node locations for $\gamma=5$.

where $r_{n}^{(\alpha, \beta) ; G R}$ comes from an $\frac{N+1}{2}$-point quadrature rule, and

$$
\Omega_{n}^{(\gamma)}= \begin{cases}\omega_{n}^{(-1 / 2, \gamma-1 / 2) ; \mathrm{GR}}, & 1 \leq n \leq \frac{N-1}{2}, \\ 2 \omega_{n}^{(-1 / 2, \gamma-1 / 2) ; \mathrm{GR}}, & n=\frac{N+1}{2}, \\ \Omega_{N+1-n}^{(\gamma)}, & \frac{N+3}{2} \leq n \leq N .\end{cases}
$$

For graphical descriptions of the above formulae, see Figure 6. By construction, when $N$ is odd, $\theta_{\frac{N+1}{2}}^{(\gamma)}=0$. The quadrature rules have no nodes at $\theta= \pm \pi$ and are symmetric rules for any $\gamma$. Thus they are always exact for any odd function. The following result holds:

Proposition 3.3. For $N$ even, the $N$-point quadrature rule $\left\{\theta_{n}^{(\gamma)}, \Omega_{n}^{(\gamma)}\right\}_{n=1}^{N}$ satisfies

$$
\int_{-\pi}^{\pi} e^{i k \theta} w_{\theta}^{(\gamma, 0)} \mathrm{d} \theta=\sum_{n=1}^{N} e^{i k \theta_{n}^{(\gamma)}} \Omega_{n}^{(\gamma)}, \quad|k| \leq N-2 .
$$

When $N$ is odd, the quadrature rule satisfies

$$
\int_{-\pi}^{\pi} e^{i k \theta} w_{\theta}^{(\gamma, 0)} \mathrm{d} \theta=\sum_{n=1}^{N} e^{i k \theta_{n}^{(\gamma)}} \Omega_{n}^{(\gamma)}, \quad|k| \leq N-1 .
$$

The degeneracy in the quadrature rule for $N$ even is of the same nature as the degeneracy in the canonical equispaced Fourier quadrature rule for an even number of grid points [14. If $\gamma=0$, the rule $\left\{\theta_{n}^{(0)}, \Omega_{n}^{(0)}\right\}_{n=1}^{N}$ is identical to the equispaced Fourier quadrature rule, symmetric about $\theta=0$. The quadrature rule $\left\{\theta_{n}^{(0)}, \Omega_{n}^{(0)}\right\}_{n=1}^{N}$ can be used to integrate against the weight function $w_{\theta}^{(\gamma, 0)}$ when $\gamma \in \mathbb{N}$ since in this case the weight is itself a trigonometric polynomial.

In order to determine a quadrature rule to integrate the weighted functions $\psi_{k}^{(\gamma)}(\theta)$, we can augment the weights $\Omega_{n}^{(\gamma)}$ to contain information about the weight function. This can be summed up in the following result: 
Corollary 3.4. The even $N$-point quadrature rule $\left\{\theta_{n}^{(\gamma)}, \omega_{n}^{(\gamma)}\right\}_{n=1}^{N}$, where $\omega_{n}^{(\gamma)}:=$ $w_{\theta}^{(-\gamma, 0)}\left(\theta_{n}^{(\gamma)}\right) \Omega_{n}^{(\gamma)}$ satisfies

$$
\int_{-\pi}^{\pi} \psi_{k}^{(\gamma)} \overline{\psi_{l}^{(\gamma)}} \mathrm{d} \theta=\sum_{n=1}^{N} \psi_{k}^{(\gamma)}\left(\theta_{n}^{(\gamma)}\right) \overline{\psi_{l}^{(\gamma)}}\left(\theta_{n}^{(\gamma)}\right) \omega_{n}^{(\gamma)}, \quad|k|+|l| \leq N-1 .
$$

Multiplying $\Omega_{n}^{(\gamma)}$ by the inverse of the weight $w_{\theta}^{(-\gamma, 0)}$ is mathematically not a problem since none of the $\theta_{n}^{(\gamma)}$ are equal to $\pm \pi$, where the weight $w_{\theta}^{(-\gamma, 0)}$ is singular. Note that since the functions $\Phi_{k}^{(s)}(x)$ are just a mapping of the functions $\Psi_{k}^{(\gamma)}(\theta)$, the quadrature rule $\left\{x\left(\theta_{n}^{(s-1)}\right), \Omega_{n}^{(s-1)}\right\}_{n=1}^{N}$, which has nodal values over $\mathbb{R}$, can be used to integrate the functions $\Phi_{k}^{(s)}(x)$ over the real line. Similarly, the rule $\left\{x\left(\theta_{n}^{(s-1)}\right), \omega_{n}^{(s-1)}\right\}_{n=1}^{N}$ can be used to integrate inner products of the generalized Wiener functions $\phi_{k}^{(s)}(x)$ over the real line.

For various $\gamma / s$ we graphically depict the location of the quadrature nodes for $N=21$ in Figure 7 on the unit circle $z \in \mathbb{T}$ and on the real line. As we increase $\gamma$ the quadrature nodes become more and more concentrated towards $z=1(\theta=0)$. On the real line, this manifests itself as higher concentration near $x=0$ which, although rectifiable via an affine mapping, is suboptimal if one wishes to capture structure away from $x=0$. The tendency of Jacobi-Gauss nodes to become more equidistant on $[-1,1]$ as $\beta$ (i.e. $\gamma$ or $s$ ) is increased 14 also suggests that these generalized quadrature rules for large $\gamma$ or $s$ will not be as accurate as the ones for smaller $\gamma$ or $s$. In addition, when $\gamma=0$, we can use these ( $\theta$-equidistant) quadrature nodes to employ the FFT for modal-nodal transformations.

3.4. The stiffness matrix. In many applications to differential equations it is necessary to express the derivative of a basis function as a linear combination of basis functions. Such an operation can be implemented via the stiffness matrix, with entries defined as:

$$
S_{k, l}^{\phi}=\left\langle\phi_{k}^{(s)}, \frac{\mathrm{d}}{\mathrm{d} x} \phi_{l}^{(s)}\right\rangle .
$$

For the generalized Wiener rational functions, the following result holds:

Theorem 3.5. Let $S^{\phi}$ denote the $N \times N$ stiffness matrix for the generalized Wiener rational functions $\phi_{k}^{(s)}$. $S^{\phi}$ satisfies the following properties for any $s>\frac{1}{2}$ :

(1) $S^{\phi}$ is skew-Hermitian, i.e., $S_{k, l}^{\phi}=-\overline{S_{l, k}^{\phi}}$

(2) $S^{\phi}$ is sparse with entries only on the super-, sub-, and main sinister and dexter diagonals: define

$$
k^{\vee}:=k-\operatorname{sgn}(k), \quad \quad k^{\wedge}:=k+\operatorname{sgn}(k) .
$$

Then

$$
\frac{\mathrm{d} \phi_{k}^{(s)}(x)}{\mathrm{d} x}=\sum_{l \in\left\{ \pm k^{\vee}, \pm k, \pm k^{\wedge}\right\}} \tau_{k, l}^{(s)} \phi_{l}^{(s)}(x),
$$

for some purely imaginary constants $\tau_{k, l}^{(s)}$. 


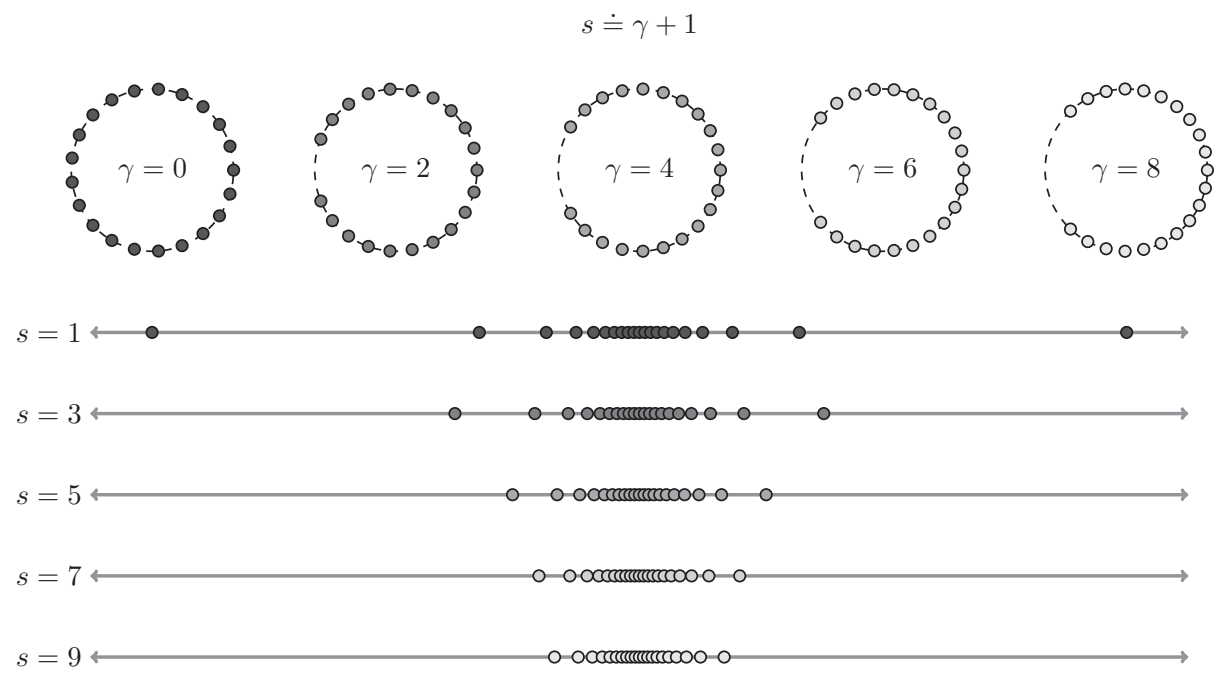

Figure 7. (Top) Plots of the Fourier quadrature nodes on the unit circle generated with (3.10), $N=21$. (Bottom) The resulting quadrature nodes on the real line. The scale on the real line is $|x| \leq 15$.

(3) The spectral radius of $S^{\phi}$ satisfies

$$
\rho\left(S^{\phi}\right) \leq N+5 s .
$$

The proof of Theorem 3.5 is quite tedious, so we only sketch the main points. Additional details are given in Appendix B.

Proof. Property 1 can easily be deduced by using integration by parts and noting that $\phi_{k}^{(s)}(x)$ decay to zero as $|x| \rightarrow \infty$.

Property 2 is provable using several properties of Jacobi polynomials. We refer the reader to [20]. The main difficulties are overcomed with the following fortuitous relations: first, that $\frac{\mathrm{d} x(\theta)}{\mathrm{d} \theta}(\theta)=(1+\cos \theta)^{-1}$, i.e., that the map we have chosen to take $\theta \rightarrow x$ has a Jacobian with a particular form; second, that

$$
\frac{\mathrm{d}}{\mathrm{d} \theta}\left[(\sin \theta) \tilde{P}_{n}^{(\alpha+1, \beta+1)}(\cos \theta)\right]
$$

is a sparse combination of $\tilde{P}_{n}^{(\alpha, \beta+1)}(\cos \theta)$.

Property 3 can be derived from the second property. The key ingredient is Gerschgorin's Theorem and the explicit entries for the constants $\tau_{k, l}^{(s)}$ given in Theorem B.3 of Appendix B.

Remark 3.6. While the $\mathcal{O}(N)$ maximum eigenvalue does depend on $s$, the proportionality factor is empirically around 2, not 5 as given in the theorem. See Table 2 , It is also worth mentioning that when $N$ is even, the stiffness matrix is invertible.

The sparsity pattern of the stiffness matrix associated with these functions (property 2 of the above theorem) is illustrated in Figure 8 . Note that the unweighted functions $\Phi^{(s)}(x)$ have a similar sparsity result; see Lemma B.2. However, the 

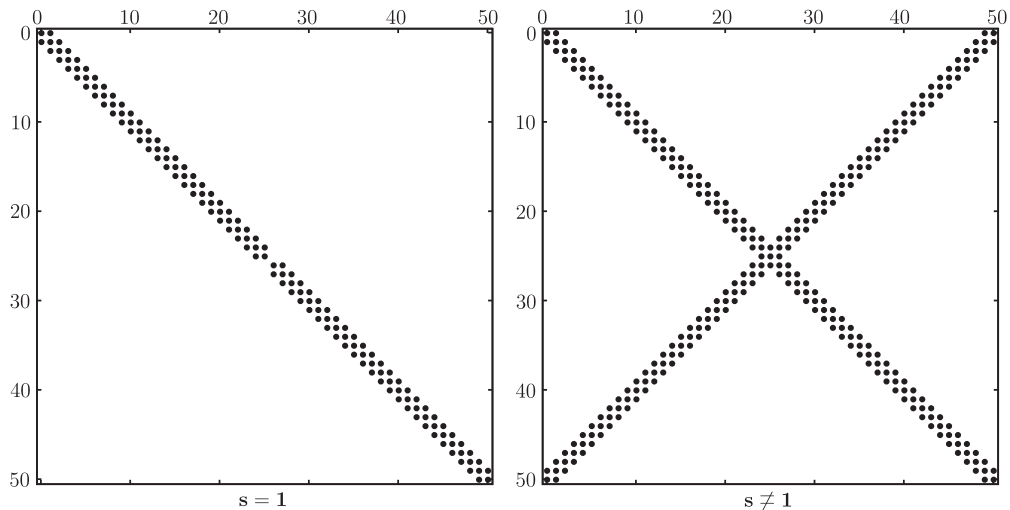

FiguRE 8. Sparsity plots for stiffness matrices of the weighted Wiener rational functions $\phi_{k}^{(s)}$. The sparsity patterns are representative of property 2 in Theorem 3.5 for $s=1$ (left) and all $s \neq 1$ (right). The $s=1$ sparsity pattern has been derived previously [11.

TABle 2. Maximum eigenvalue of the $N \times N$ stiffness matrix $S^{\phi}$ for the Wiener rational functions $\phi_{k}^{(s)}$. The results adhere to the asymptotic bound given in property 3 of Theorem 3.5 .

\begin{tabular}{|r|c|c|c|c|c|}
\hline$s \backslash \mathrm{N}$ & 11 & 50 & 101 & 250 & 501 \\
\hline 0.6 & 7.31 & 43.76 & 91.50 & 237.60 & 483.75 \\
\hline 1.0 & 7.99 & 44.51 & 92.28 & 238.39 & 484.54 \\
\hline 6.0 & 15.96 & 53.75 & 101.81 & 248.14 & 494.40 \\
\hline$\pi^{2} \approx 9.87$ & 21.72 & 60.67 & 109.05 & 255.63 & 501.99 \\
\hline 15.5 & 29.73 & 70.45 & 119.40 & 266.44 & 512.99 \\
\hline
\end{tabular}

Fourier functions $\Psi^{(\gamma)}(\theta)$ and $\psi^{(\gamma)}(\theta)$ do not have sparse stiffness matrices (unless $\gamma=0$ ). In addition, numerical values for the maximum eigenvalues of the stiffness matrix (property 3 ) are given in Table 2

\section{THE SEMI-INFINITE INTERVAL}

The generalized Wiener basis functions can be used for function expansions on the infinite line. For expansions on semi-infinite intervals, we can instead use either the even or odd Jacobi polynomial basis sets that make up the Fourier functions constructed in Section 2.3. We obain the collection of functions

$$
\begin{aligned}
\rho_{n}^{(s)} & =\sqrt{w_{x}^{(s, 0)}} \Phi_{n}^{(s)}(x) \\
& =\left(\frac{2}{x^{2}+1}\right)^{s / 2} \tilde{P}_{n}^{(-1 / 2, s-3 / 2)}\left(\frac{1-x^{2}}{1+x^{2}}\right), \quad n \in \mathbb{N}_{0} .
\end{aligned}
$$

These functions are a direct mapping and weighting of the Jacobi polynomials. Because of this, they are orthonormal and complete in $L^{2}((0, \infty), \mathbb{R})$. Mapping 
techniques for classical functions are not novel and we briefly discuss existing methods in Section 5 in particular, the popular mapping $r=\frac{1-x}{1+x}$ is addressed in Section 5.2 .

We make use of the regular square root function $\sqrt{w_{x}^{(s, 0)}}$ in (4.1) instead of the phase-shifted version $\sqrt[*]{w_{x}^{(s, 0)}}$ because there is no need to have complex-valued functions.

The caveat in using these functions for expansions on the semi-infinite interval is the fact that they all have zero-valued odd derivatives at $x=0$. This parallels the property at $\theta=0$ for a cosine series on $\theta \in[0, \pi]$.

The restriction of the Wiener functions to the semi-infinite interval as defined in (4.1) comes both with advantages and sacrifices. A numerical comparison between those mapping techniques, the functions (4.1), and the Laguerre functions will be made in Part II.

\section{Connection to Alternative methods}

Finally, we briefly discuss existing methods in the literature and their relation to the Wiener rational basis. Numerical studies comparing these methods are presented in Part II.

5.1. The infinite interval. The Wiener basis functions are the result of mapping a classical basis set to fit a non-classical interval [13]. One of the more popular mappings that has gained momentum in the literature results in the so-called 'mapped Chebyshev' functions/polynomials.

In order to further generalize the mapped Chebyshev functions, we will briefly restate their derivation. From the Jacobi polynomials $P_{n}^{(\alpha, \beta)}(r(\theta))$ defined on $\theta \in$ $[0, \pi]$, we 'stretch' the domain to $\Theta \in[-\pi, \pi]$ via the affine mapping $\Theta=2 \theta-\pi$. Finally, we utilize the linear fractional map $z=\frac{i-x}{i+x}$ to yield functions on the real line $x \in \mathbb{R}$. For all $s, t>\frac{1}{2}$, this results in the functions $\mathrm{PB}_{n}^{(s, t)}(x)$, defined as

$$
\mathrm{PB}_{n}^{(s, t)}(x)=\widetilde{P}_{n}^{((2 s-3) / 2,(2 t-3) / 2)}\left(\frac{x}{\sqrt{1+x^{2}}}\right),
$$

orthonormal on the real line under the weight

$$
w_{\mathrm{PB}}^{(s, t)}=\left[1-\frac{x}{\sqrt{1+x^{2}}}\right]^{(2 s-3) / 2}\left[1+\frac{x}{\sqrt{1+x^{2}}}\right]^{(2 t-3) / 2},
$$

and the orthonormal weighted functions

$$
\mathrm{pb}_{n}^{(s, t)}:=\sqrt{w_{\mathrm{PB}}^{(s, t)}} \mathrm{PB}_{n}^{(s, t)},
$$

When $s=t=1$, the functions $\mathrm{PB}_{n}^{(s, t)}$ coincide with the mapped Chebyshev polynomials $\operatorname{TB}_{n}(x)$ introduced in [4] and subsequently developed in [7] and [9]. The mapped Jacobi functions $\mathrm{pb}_{n}^{(s, t)}$ decay like $\frac{1}{|x|^{s}}$ for $x \rightarrow-\infty$ and $\frac{1}{|x|^{t}}$ for $x \rightarrow+\infty$. The advantage of these functions is that the decay can be different as $x \rightarrow \pm \infty$; they enjoy established convergence theory in function spaces [3] and applications to differential equations 28 for the Chebyshev case $s=t=1$. In Part II we will consider the basis set $\mathrm{pb}_{n}^{(s, t)}$ with $s=t=1$, i.e., the Chebyshev case.

Note that because all of these mapped polynomials and the generalized Wiener basis both ultimately stem from Jacobi polynomials and mappings of a similar 
TABLE 3. Relationship between orthogonal functions in previous work and the current bases presented.

\begin{tabular}{|c|c|c|c|c|}
\hline Previous function & Name/classification & Interval & Reference & Relation \\
\hline$T B_{n}$ & Cheyshev rational functions (1st) & $\mathbb{R}$ & {$[8],[4],[8]$} & $\mathrm{PB}_{n}^{(1,1)}$ \\
\hline$S B_{n} / U B_{n}$ & Chebyshev rational functions (2nd) & $\mathbb{R}$ & {$[8],[8],[9]$} & $\mathrm{PB}_{n}^{(2,2)}$ \\
\hline$C_{n} / \mathrm{CC} n$ & Christov functions (even) & $\mathbb{R},[0, \infty)$ & {$[1],[8]$} & $\operatorname{Im}\left\{\phi_{n}^{(1)}\right\}$ \\
\hline$S_{n} / \mathrm{SC}_{n}$ & Christov functions (odd) & $\mathbb{R},[0, \infty)$ & {$[1],[8]$} & $\operatorname{Re}\left\{\phi_{n}^{(1)}\right\}$ \\
\hline$C H_{n}$ & Higgins functions (even) & $\mathbb{R},[0, \infty)$ & {$[8]$} & $\operatorname{Re}\left\{\Phi_{n}^{(1)}\right\}$ \\
\hline$S H_{n}$ & Higgins functions (odd) & $\mathbb{R},[0, \infty)$ & {$[8]$} & $\operatorname{Im}\left\{\Phi_{n}^{(1)}\right\}$ \\
\hline$\rho_{k}$ & (Complex) Higgins functions & $\mathbb{R}$ & {$[15,[1]$} & $\Phi_{k}^{(1)}$ \\
\hline$\sigma_{k}$ & (Complex) Wiener rational functions & $\mathbb{R}$ & {$[27,[1]$} & $\phi_{k}^{(1)}$ \\
\hline$T L_{n}$ & Semi-infinte Chebyshev rational functions & {$[0, \infty)$} & {$[6]$} & $\mathrm{PL}_{n}^{(1 / 2)}$ \\
\hline
\end{tabular}

character, these basis sets can be expected to be related in some fashion. For example, to relate the mapped Jacobi functions to the generalized Wiener rational functions, we have

$$
\operatorname{PB}_{n}^{(s, s)}(x) \propto \operatorname{Re}\left\{\Phi_{n}^{(s)}\left(\frac{x+\sqrt{x^{2}+1}-1}{x-\sqrt{x^{2}+1}+1}\right)\right\} .
$$

In Table 3 we relate the unweighted functions to the generalized Wiener rational basis, modulo multiplicative constants. In this article we make no observations about how mapped Jacobi polynomials compare to the Wiener basis set as a practical tool for function expansions. However, such a comparison will be a central theme in Part II.

5.2. The semi-infinite interval. The mapping technique for the semi-infinite interval is analogous to the infinite interval case. We use the popular mapping

$$
x=\frac{1-r}{1+r}, \quad r=\frac{1-x}{1+x},
$$

which again is a Möbius map. If necessary, one can specify the relationship to $\theta$ and the cosine series on $[0, \pi]$. For details, see [6]. Our definition of the transformation differs only in orientiation from that presented in 6]. Approximation theory for this mapping is given in 22. We have chosen this orientation so that the Jacobi parameter $\beta$ is assigned to the location $x=\infty$ in order to mimic the same assignment for the Wiener functions on infinite intervals.

In the literature the maps of the Chebyshev polynomials under the transformation (5.1) are labeled $\mathrm{TL}_{n}(x)$. Adopting similar notation, we define

$$
\mathrm{PL}_{n}^{(s)}(x)=\tilde{P}_{n}^{(-1 / 2,2 s-3 / 2)}\left(\frac{1-x}{1+x}\right), \quad x \in[0, \infty],
$$


which are $L^{2}$-complete and orthonormal under the weight function

$$
w_{\mathrm{PL}}^{(s)}(x)=\frac{1}{2 \sqrt{x}}\left(\frac{2}{1+x}\right)^{(2 s)} .
$$

It is then possible to define the weighted functions

$$
\begin{aligned}
\operatorname{pl}_{n}^{(s)}(x) & =\left(\frac{2}{1+x}\right)^{s} \mathrm{PL}_{n}^{(s)}(x) \\
& =\left(\frac{2}{1+x}\right)^{s} \tilde{P}_{n}^{(-1 / 2,2 s-2)}\left(\frac{1-x}{1+x}\right),
\end{aligned}
$$

which are $L^{2}$-complete and orthonormal under the weighted $L^{2}$ inner product

$$
\langle f, g\rangle_{w_{P L}^{(0)}}=\int_{0}^{\infty} f g \frac{1}{2 \sqrt{x}} \mathrm{~d} x,
$$

for any $s>\frac{1}{2}$. The $\mathrm{pl}_{n}^{(s)}$ are defined for $x \in[0, \infty]$ and decay like $x^{s}$ as $x \rightarrow \infty$. We use the weighted inner product above in order to ensure that integer values of $s$ are amenable to the FFT. These functions inherit the $\mathcal{O}\left(N^{2}\right)$ time-step restriction for explicit nodal time-integration methods for time-dependent PDEs. The same observation can be made about the functions defined in [6].

\section{Conclusion}

We have presented a collection of generalized Fourier series which, when mapped and weighted appropriately, generates a basis set on the infinite interval with a tunable rate of decay. For each rate of decay $s$ satisfying $s>\frac{1}{2}$ the resulting basis set $\phi_{k}^{(s)}$ :

- is orthonormal and complete in $L^{2}(\mathbb{R}, \mathbb{C})$,

- is characterized by $x^{-s}$ decay for $|x| \rightarrow \infty$,

- can be generated via Jacobi polynomial recurrence relations,

- has sparse connection properties that can be efficiently exploited via combinations of sparse Fourier and Jacobi connections,

- has an $N \times N$ Galerkin stiffness/differentiation matrix that has at most $6 N$ non-zero entries with $\mathcal{O}(N)$ spectral radius,

- is characterized by a 'Gauss-like' quadrature rule.

When $s \in \mathbb{N}$, the basis set is a rational function; we will show in Part II that in this case we can use the FFT for modal-nodal transformations. The case $s=1$ corresponds to a mapping and weighting of the canonical Fourier series, as discussed by others previously. Due to the original presentation of the $s=1$ basis by Wiener [27, we choose to call the functions $\phi_{k}^{(s)}$ the generalized Wiener rational basis functions.

These basis functions have a similar flavor to directly mapped and weighted Jacobi polynomials (called $\mathrm{pb}_{n}^{(s, t)}$ here). In Part II we will compare these basis sets and discuss advantages and disadvantages of each. In addition, we will also employ the Sinc and Hermite functions in test cases in an attempt to investigate a relatively broad class of spectral approximation methods. In contrast to [23] which reviews much of the theory present for expansions on the infinite interval, we concentrate on numerical issues, including application of the FFT. We will extend our investigation to the semi-infinite interval to compare the Laguerre polynomials/functions, the 
mapped Jacobi functions (denoted $\mathrm{pl}_{n}^{(s)}$ here), and the restriction of the Wiener functions to the semi-infinite interval as given in Section 4

\section{Appendix A. Recurrence coefficients}

In this appendix we compile recurrence relations for the Jacobi/Szegö-Fourier/ Wiener functions. We state the recurrences in terms of the Szegö-Fourier functions $\Psi_{k}^{(\gamma)}$, but note that they all apply equally well to the unweighted Wiener rational functions. We only list recurrences for $k \geq 0$; for $k<0$, we may use complex conjugation to obtain $\Psi_{-|k|}^{(\gamma)}$ at no additional cost. We first require a tour of some Jacobi polynomial recurrences:

$$
\begin{aligned}
\sqrt{b_{n+1}^{(\alpha, \beta)}} \tilde{P}_{n+1}^{(\alpha, \beta)} & =\left[r-a_{n}^{(\alpha, \beta)}\right] \tilde{P}_{n}^{(\alpha, \beta)}-\sqrt{b_{n}^{(\alpha, \beta)}} \tilde{P}_{n-1}^{(\alpha, \beta)} \\
\left(1-r^{2}\right) \tilde{P}_{n}^{(\alpha, \beta)} & =\sum_{i=0}^{2} \varepsilon_{n, i}^{(\alpha, \beta)} \tilde{P}_{n+i}^{(\alpha-1, \beta-1)} \\
\tilde{P}_{n}^{(\alpha, \beta)} & =\sum_{i=0}^{2} \eta_{n,-i}^{(\alpha, \beta)} \tilde{P}_{n-i}^{(\alpha+1, \beta+1)}
\end{aligned}
$$

The three-term recurrence coefficients in (A.1) are found, e.g., in [12. The recurrence coefficients in (A.2) can be obtained by determining the analogous relations for the monic orthogonal polynomials ([1, 24]) and then employing the appropriate normalizations:

$$
\begin{aligned}
& \varepsilon_{n, 0}^{(\alpha, \beta)}= \begin{cases}2 \sqrt{\frac{\alpha \beta}{(\alpha+\beta)(\alpha+\beta+1)}}, & n=0, \\
\frac{2}{(\alpha+\beta+2)} \sqrt{\frac{(\alpha+1)(\beta+1)(\alpha+\beta)}{(\alpha+\beta+3)}}, & n=1, \\
\frac{2}{(2 n+\alpha+\beta)} \sqrt{\frac{(n+\alpha)(n+\beta)(n+\alpha+\beta-1)(n+\alpha+\beta)}{(2 n+\alpha+\beta-1)(2 n+\alpha+\beta+1)}}, & n>1 .\end{cases} \\
& \varepsilon_{n, 1}^{(\alpha, \beta)}= \begin{cases}\frac{2(\alpha-\beta)}{(\alpha+\beta+2) \sqrt{\alpha+\beta}} & n=0, \\
\frac{2(\alpha-\beta) \sqrt{(n+1)(n+\alpha+\beta)}}{(2 n+\alpha+\beta)(2 n+\alpha+\beta+2)}, & n>0 .\end{cases} \\
& \varepsilon_{n, 2}^{(\alpha, \beta)}= \begin{cases}\frac{2}{\alpha+\beta+2} \sqrt{\frac{2(\alpha+1)(\beta+1)}{(\alpha+\beta+1)(\alpha+\beta+3)}}, & n=0, \\
\frac{2}{2 n+\alpha+\beta+2} \sqrt{\frac{(n+1)(n+2)(n+\alpha+1)(n+\beta+1)}{(2 n+\alpha+\beta+1)(2 n+\alpha+\beta+3)}}, & n>0 .\end{cases}
\end{aligned}
$$

Using the orthogonal polynomial three-term recurrence relation (A.1) we can show the following recurrence relation for the Szegö-Fourier functions $\Psi_{n}^{(\gamma)}(\theta)$ :

$$
\begin{aligned}
\Psi_{n+1}^{(\gamma)}= & {\left[U_{n}^{(\gamma)} \cos \theta-V_{n}^{(\gamma)}\right] \Psi_{n}^{(\gamma)}+\left[U_{-n}^{(\gamma)} \cos \theta-V_{-n}^{(\gamma)}\right] \Psi_{-n}^{(\gamma)} } \\
& -W_{n}^{(\gamma)} \Psi_{n-1}^{(\gamma)}-W_{-n}^{(\gamma)} \Psi_{-(n-1)}^{(\gamma)} .
\end{aligned}
$$

Given $\gamma>-\frac{1}{2}$ we assign the values $\alpha=-\frac{1}{2}$ and $\beta=\gamma-\frac{1}{2}$. Using the three-term recurrences (A.2)-(A.3) we have:

$$
\begin{aligned}
\Psi_{n+1}^{(\gamma)}= & {\left[\tilde{U}_{n}^{(\gamma)} i \sin \theta-\tilde{V}_{n}^{(\gamma)}\right] \Psi_{n}^{(\gamma)}+\left[\tilde{U}_{-n}^{(\gamma)} i \sin \theta-\tilde{V}_{-n}^{(\gamma)}\right] \Psi_{-n}^{(\gamma)} } \\
& -\tilde{W}_{n}^{(\gamma)} \Psi_{n-1}^{(\gamma)}-\tilde{W}_{-n}^{(\gamma)} \Psi_{-(n-1)}^{(\gamma)},
\end{aligned}
$$


Putting these last two recurrences together yields

$$
\begin{aligned}
D_{n}^{(\gamma)} \Psi_{n+1}^{(\gamma)}=[ & \left.A_{n}^{(\gamma)} e^{i \theta}-B_{n}^{(\gamma)}\right] \Psi_{n}^{(\gamma)}+\left[A_{-n}^{(\gamma)} e^{-i \theta}-B_{-n}^{(\gamma)}\right] \Psi_{-n}^{(\gamma)} \\
& +C_{n}^{(\gamma)} \Psi_{n-1}^{(\gamma)}+C_{-n}^{(\gamma)} \Psi_{-(n-1)}^{(\gamma)},
\end{aligned}
$$

with the following values for the recurrence coefficients:

$$
\begin{aligned}
& D_{n}^{(\gamma)}= \begin{cases}4 \varepsilon_{0,2}^{(\alpha, \beta)} \sqrt{\gamma}, & n=0, \\
2 \varepsilon_{n, 2}^{(\alpha, \beta)}[\sqrt{n+\gamma}+\sqrt{n}, & n>0,\end{cases} \\
& A_{ \pm n}^{(\gamma)}= \begin{cases}\sqrt{2}[\sqrt{\gamma+1} \pm 1], & n=0, \\
\sqrt{n+\gamma+1} \pm \sqrt{n+1}, & n>0,\end{cases} \\
& B_{ \pm n}^{(\gamma)}= \begin{cases}\frac{2 \gamma \sqrt{2}}{\sqrt{\gamma+1}}, & n=0, \\
\frac{-\varepsilon_{n, 1}^{(\alpha, \beta)}}{2 \sqrt{(n+1)(n+\gamma-1)}}\left(\gamma A_{ \pm n}^{(\gamma)}+[2 \sqrt{n(n+\gamma)}-1] A_{\mp n}^{(\gamma)}\right), & n>0,\end{cases} \\
& C_{ \pm n}^{(\gamma)}= \begin{cases}0, & n=0, \\
\frac{1}{A_{0}^{(\gamma)}(\gamma+1)} \sqrt{\frac{(2 \gamma+1) \gamma}{\gamma+2}}, & n=1, \\
\frac{\gamma \varepsilon_{n, 0}^{(\alpha, \beta)}}{\sqrt{(n+\gamma-2)(n+\gamma-1)} A_{n-1}^{(\gamma)}}\left[\sqrt{(n+\gamma)^{2}-1}-\sqrt{n^{2}-1}\right], & n>1 .\end{cases}
\end{aligned}
$$

Appendix B. The Stiffness matrix

We assume the decay parameter $s>\frac{1}{2}$ is given and we derive $\alpha$ and $\beta$ from the value $\gamma:=s-1$ as in Appendix $\mathrm{A}$. Also, we define increments and decrements of the integer index $k \in \mathbb{Z}$ :

$$
k^{\vee}=\operatorname{sgn}(k)(|k|-1), \quad k^{\wedge}=\operatorname{sgn}(k)(|k|+1), \quad n:=|k|-1 .
$$

We begin by noting the sparse representation of the product of $\phi_{k}^{(s)}$ and $\frac{1}{(x-i)}$ :

Lemma B.1. We have the representation:

$$
\frac{-s}{(x-i)} \phi_{k}^{(s)}=\sum_{l \in\left\{ \pm k^{\vee}, \pm k, \pm k^{\wedge}\right\}} \chi_{k, l}^{(s)} \phi_{l}^{(s)},
$$

for some constants $\chi_{k, l}^{(s)}$.

Proof. We first note that

$$
\frac{-s}{x-i}=-\frac{s}{2}[\sin \theta(x)+i(1+\cos \theta(x))],
$$

after making the transformation to $\theta(x)$. Then making the identification $\Phi_{k}^{(s)}=$ $\Psi_{k}^{(s-1)}$, we may use recurrence relations (A.4)- (A.5) to obtain the result.

A second more potent result is the sparsity result for the unweighted Wiener rational functions $\Phi_{k}^{(s)}(x)$ :

Lemma B.2. We have

$$
\frac{\mathrm{d} \Phi_{k}^{(s)}(x)}{\mathrm{d} x}=\sum_{l \in\left\{ \pm k^{\vee}, \pm k, \pm k^{\wedge}\right\}} \sigma_{k, l}^{(s)} \Phi_{l}^{(s)},
$$

where the $\sigma_{k, l}^{(s)}$ are constants. 
Proof. This result can be obtained by brute-force calculation of the derivative along with some Jacobi polynomial relations. Two critical steps are necessary: a collapsing of a special arithmetic combination involving various constants in several Jacobi polynomial relations, and the form of the $\theta \rightarrow x$ Jacobian for the mapping. Thus, the particular form of the mapping is critical in proving this result.

Putting the two lemmas together, we have the desired sparsity result for the $\phi_{k}^{(s)}(x)$ stiffness matrix:

Theorem B.3. The following equality holds for any $s>\frac{1}{2}$ :

$$
\frac{\mathrm{d} \phi_{k}^{(s)}}{\mathrm{d} x}=\sum_{l \in\left\{ \pm k^{\vee}, \pm k, \pm k^{\wedge}\right\}} \tau_{k, l}^{(s)} \phi_{l}^{(s)},
$$

where the constants $\tau_{k, l}^{(s)}$ are equal to

$$
\begin{aligned}
\tau_{k, \pm k^{\vee}}= & \frac{i}{4} \sqrt{1-\frac{s(s-2)}{(2 n+s-1)(2 n+s+1)}} \\
& \times\{\operatorname{sgn}(k)(\sqrt{(n+s-1)(n+s)} \pm \sqrt{n(n+1)}) \\
& \left.+\frac{-s}{(2 n+s)}(\sqrt{(n+1)(n+s-1)} \pm \sqrt{n(n+s)})\right\}, \\
\tau_{k, k}= & i \operatorname{sgn}(k) \sqrt{(n+1)(n+s)}-\frac{i s(s-1)^{2}}{2(2 n+s)(2 n+s+2)}-\frac{i s}{2}, \\
\tau_{k,-k}= & \frac{i s(s-1)}{2(2 n+s)(2 n+s+2)}, \\
\tau_{k, \pm k^{\wedge}}= & \frac{i}{4} \sqrt{1-\frac{s(s-2)}{(2 n+s+1)(2 n+s+3)}} \\
& \times\left\{-\frac{s}{2 n+s+2}[\sqrt{(n+2)(n+s)} \pm \sqrt{(n+1)(n+s+1)}]\right. \\
& +\operatorname{sgn}(k)[\sqrt{(n+1)(n+2)} \pm \sqrt{(n+s)(n+s+1)}]\} .
\end{aligned}
$$

Clearly for $|k|=1$ we have $\tau_{k, \pm k^{\vee}}=\tau_{k, 0}$. Taking into account the different normalization constant in the definition of $\phi_{k}^{(s)}$ for $|k|=1$ we have:

$$
\tau_{k, 0}=\frac{i}{2} \sqrt{\frac{2 s-1}{2 s+2}}\{\operatorname{sgn}(k) \sqrt{s}-1\}, \quad|k|=1 .
$$

For $k=0$ :

$$
\frac{\mathrm{d} \phi_{0}^{(s)}}{\mathrm{d} x}=-\frac{i \sqrt{s-\frac{1}{2}}}{2}\left[\frac{1+\sqrt{s}}{\sqrt{1+s}} \phi_{-1}^{(s)}+2 \sqrt{s-\frac{1}{2}} \phi_{0}^{(s)}+\frac{1-\sqrt{s}}{\sqrt{1+s}} \phi_{1}^{(s)}\right] .
$$

Proof. The result of this theorem follows from a simple application of the product rule of differentiation to

$$
\frac{\mathrm{d}}{\mathrm{d} x}\left[\frac{2^{s / 2}}{(x-i)^{s}} \Phi_{k}^{(s)}\right],
$$

with the appropriate use of Lemmas B.1 and B.2. 
With explicit entries for the stiffness matrix derived, we are now ready to give the proof of the third property of Theorem 3.5 , the spectral radius of the stiffness matrix.

Proof. We can crudely bound the entries of the stiffness matrix for $n:=|k|-1>1$ :

$$
\begin{array}{lll}
\left|\tau_{k, k^{\vee}}\right| \leq \frac{n}{2}+s, & \left|\tau_{k,-k^{\vee}}\right| \leq \frac{s}{2}, \\
\left|\tau_{k, k}\right| \leq n+2 s, & \left|\tau_{k,-k}\right| \leq 1, \\
\left|\tau_{k, k^{\wedge}}\right| \leq \frac{n}{2}+s+\frac{1}{2}, & \left|\tau_{k,-k^{\wedge}}\right| \leq \frac{s}{4} .
\end{array}
$$

Thus we have:

$$
\begin{aligned}
\left|\tau_{-k}\right|+\left|\tau_{k^{\vee}}\right|+\left|\tau_{-k^{\vee}}\right|+\left|\tau_{k^{\wedge}}\right|+\left|\tau_{-k^{\wedge}}\right| & \leq 1+\frac{n}{2}+s+\frac{s}{2}+\frac{n}{2}+s+\frac{1}{2}+\frac{s}{4} \\
& <n+3 s+2 .
\end{aligned}
$$

An application of Gerschgorin's Theorem proves the result.

\section{ACKNOWLEDGMENTS}

The authors acknowledge partial support for this work by AFOSR award FA955007-1-0422. The authors are indebted to the anonymous reviewers whose helpful suggestions and constructive comments greatly improved the quality of this paper.

\section{REFERENCES}

1. M. Abramowitz and I. Stegun, Handbook of mathematical functions, Dover, 1972.

2. R. Askey, Orthogonal polynomials and special functions, SIAM, 1975. MR0481145 (58:1288)

3. G. Ben-Yu and W. Zhong-Qing, Modified Chebyshev rational spectral method for the whole line, Proceedings of the fourth international conference on dynamical systems and differential equations, 2002, pp. 365-374.

4. J.P. Boyd, The optimization of convergence for Chebyshev polynomial methods in an unbounded domain, Journal of computational physics 45 (1982), 43-79. MR650425 (83d:65073)

5. _ Asymptotic coefficients of Hermite function series, Journal of Computational Physics 54 (1984), 382-410. MR755455 (86c:41012)

6. - Orthogonal rational functions on a semi-infinite interval, Journal of Computational Physics 70 (1987), 63-88. MR888932 (88d:65034)

7. _ Spectral methods using rational basis functions on an infinite interval, Journal of Computational Physics 69 (1987), 112-142. MR892255 (88e:65093)

8. $\quad$ The orthogonal rational functions of Higgins and Christov and algebraically mapped Chebyshev polynomials, Journal of Approximation Theory 61 (1990), 98-105. MR.1047151 (91c:41039)

9. - Chebyshev and Fourier spectral methods, second ed., Dover, 2001. MR1874071 (2002k:65160)

10. A.B. Cain, J.H. Ferziger, and W.C. Reynolds, Discrete orthogonal function expansions for non-uniform grids using the fast Fourier transform, Journal of Computational Physics 56 (1984), 272-286. MR768478 (86e:65170)

11. C.I. Christov, A complete orthonormal system of functions in $L^{2}(-\infty, \infty)$ space, SIAM Journal of Applied Math 42 (1982), no. 6, 1337-1344. MR678221 (84b:42018)

12. W. Gautschi, Orthogonal polynomials: Computation and approximation, Numerical Mathematics and Scientific Computation, Oxford University Press, 2004. MR.2061539|(2005e:42001)

13. D. Gottlieb and S.A. Orszag, Numerical analysis of spectral methods: theory and applications, CBMS-NSF Regional Conference Series in Applied Mathematics, SIAM, 1977. MR0520152 $(58: 24983)$

14. J.S. Hesthaven, S. Gottlieb, and D. Gottlieb, Spectral methods for time-dependent problems, Monographs on applied and computational mathematics, Cambridge, 2007. MR 2333926 (2008i:65223)

15. J.R. Higgins, Completeness and basis properties of sets of special functions, Cambridge University Press, 1977. MR0499341 (58:17240) 
16. M. Klaus, D.E. Pelinovsky, and V.M. Rothos, Evans functions for Lax operators with algebraically decaying potentials, Nonlinear Science 16 (2006), 1-44. MR2202901 (2007b:37180)

17. J. Lund and K.L. Bowers, Sinc methods for quadrature and differential equations, SIAM, 1992. MR 1171217 (93i:65004)

18. E. Magyari and B. Keller, The algebraically decaying wall jet, European Journal of Mechanics B: Fluids 23 (2004), 601-605.

19. P. Maroni and Z. da Rocha, Connection coefficients between orthogonal polynomials and the canonical sequence: an approach based on symbolic computation, Numerical Algorithms 47 (2008), 291-314. MR2385739 (2009e:33028)

20. A.C. Narayan, A generalization of the Wiener rational basis functions on infinite intervals, Ph.D. thesis, Brown University, Providence, RI, 2009.

21. A.C. Narayan and J.S. Hesthaven, The application of the fast Fourier transform to Jacobi polynomial expansions, Submitted (2009).

22. Zhong qing Wang and Ben yu Guo, Jacobi rational approximation and spectral method for differential equations of degenerate type, Mathematics of Computation 77 (2008), no. 262, 883-907. MR2373184 (2008m:65274)

23. J. Shen and L.-L. Wang, Some recent advances on spectral methods for unbounded domains, Communcations in Computationals Physics 5 (2009), no. 2-4, 195-241. MR2513685

24. G. Szegö, Orthogonal polynomials, 4 ed., AMS Colloquium Publications, volume XXIII, American Mathematical Society, 1975. MR 0372517 (51:8724)

25. J.A.C. Weidemann, The eigenvalues of Hermite and rational spectral differentiation matrices, Numerische Mathematik 61 (1992), 409-431. MR.1151779 (92k:65071)

26. E.T. Whittaker, On the functions which are represented by the expansions of the interpolation theory, Proceedings of the Royal Society of Edinburgh 35 (1915), 181-194.

27. N. Wiener, Extrapolation, interpolation, and smoothing of stationary time series, MIT Technology Press and John Wiley \& Sons, 1949. MR0031213 (11:118j)

28. W. Zhong-Qing and G. Ben-Yu, A rational approximation and its applications to nonlinear partial differential equations on the whole line, Mathematical Analysis and Applications $\mathbf{2 7 4}$ (2002), 374-403. MR1936704 (2003g:41019)

Department of Mathematics, Purdue University, 150 N. University Street, West LAFAYETTE, INDIANA 47907

E-mail address: acnaraya@purdue.edu

Division of Applied Mathematics, Brown University, 182 George Street, Box F, ProvIDENCE, Rhode Island 02912

E-mail address: Jan.Hesthaven@brown.edu 